\title{
Induction of tumor apoptosis through a circular RNA enhancing Foxo3 activity
}

\author{
William W Du ${ }^{1,2,5}$, Ling Fang ${ }^{1,2,3,5}$, Weining Yang ${ }^{1,5}$, Nan Wu ${ }^{1,2}$, Faryal Mehwish Awan ${ }^{1,2}$, Zhenguo Yang ${ }^{1,2}$ and Burton B Yang ${ }^{\star 1,2,4}$
}

Circular RNAs are a class of non-coding RNAs that are receiving extensive attention. Despite reports showing circular RNAs acting as microRNA sponges, the biological functions of circular RNAs remain largely unknown. We show that in patient tumor samples and in a panel of cancer cells, circ-Fox03 was minimally expressed. Interestingly, during cancer cell apoptosis, the expression of circ-Fox03 was found to be significantly increased. We found that silencing endogenous circ-Fox03 enhanced cell viability, whereas ectopic expression of circ-Foxo3 triggered stress-induced apoptosis and inhibited the growth of tumor xenografts. Also, expression of circ-Foxo3 increased Fox03 protein levels but repressed p53 levels. By binding to both, circ-Foxo3 promoted MDM2-induced p53 ubiquitination and subsequent degradation, resulting in an overall decrease of p53. With low binding affinity to Fox03 protein, circ-Fox03 prevented MDM2 from inducing Fox03 ubiquitination and degradation, resulting in increased levels of Foxo3 protein. As a result, cell apoptosis was induced by upregulation of the Foxo3 downstream target PUMA.

Cell Death and Differentiation (2017) 24, 357-370; doi:10.1038/cdd.2016.133; published online 25 November 2016

Circular RNAs are a large class of non-coding RNAs that are circularized by joining free $3^{\prime}-$ to $5^{\prime}$-ends, forming a circular structure. ${ }^{1-4}$ Although circular RNAs were initially characterized over 30 years ago, their functions in mammalian cells are still largely unknown. Most circular RNAs are predominantly found in the cytoplasm and contain exons, known as circRNAs. ${ }^{5}$ A relatively smaller group of circular RNAs that contain both exons and introns are known as ElciRNAs, and are predominantly found in the nucleus. ${ }^{6}$ Recent studies have indicated that some circular RNAs contain miRNA binding sites and may function as sponges to arrest miRNA functions. ${ }^{7,8}$ It has further been reported that ElciRNAs increase the transcription of their parental genes. ${ }^{9}$ Recently, we showed that the circular RNA circ-Foxo3 could function by binding to proteins in related signal pathways. ${ }^{10,11}$ In the present study, we used computational approach to elucidate the interaction of circ-Foxo3 with MDM2 and p53. The RING-finger domain in the carboxyl terminal of the MDM2 is known to bind RNA specifically in a sequence-specific manner, ${ }^{12}$ whereas p53 interacts with RNA via its C-terminal regulatory domain. ${ }^{13}$ Our study comprised of computer-aided RNA structure modeling of circ-Foxo3 employing minimum free energy algorithm and machine translation system followed by its molecular interaction with MDM2 (RING-finger domain) and p53 (C-terminal regulatory domain) that includes docking, scoring, clustering, and refinement of the most promising models. The interaction was further confirmed by an approach of molecular experiments to explicate the biological functions of circ-Foxo3.

\section{Results}

Decreased expression of circ-Foxo3 in tumors and cancer cells. Downregulation of Foxo3 is often observed in cancer development. ${ }^{14,15}$ Both circ-Foxo3 and Foxo3 mRNA are encoded by the $\mathrm{FOXO} 3$ gene. ${ }^{16}$ We found that the levels of circ-Foxo3 in tumor specimen were significantly lower than in the adjacent benign tissue (Figure 1a). We examined circ-Foxo3 expression and detected significantly higher levels of circ-Foxo3 in six non-cancerous cell lines (Hek293T, BEAS2B, HaCaT, NIH3T3, MEF, and MCF-10A) than in the cancer cell lines MDA-MB-468, MDA-MB-231, 67NR, 66C14, 4T07, 4T1, and B16 (Figure 1b).

Using real-time PCR, we found that circ-Foxo3 was expressed at significantly higher levels in the cell lines 66C14, 4T1, MDA-MB-468, and MDA-MB-231, when the cells were treated with $\mathrm{H} 2 \mathrm{O} 2$ (Figure 1c), Doxorubicin (Figure 1d), and Cisplatin (Figure 1e). This was consistent with the increased cell death (Figure 1f, Supplementary Figure S1a) and upregulation of Bax and Puma and cleaved Caspase-3 (Figure 1g). We analyzed cell cycle distribution and confirmed that treatment with these stressors increased cell number in the $\mathrm{G} 1$ phase (Figure $1 \mathrm{~h}$, Supplementary Figure S1b).

To explore the effect of circ-Foxo3 on apoptosis, we designed siRNA specifically targeting circ-Foxo3. Transfection with this siRNA in cancer cell line 4T1 successfully decreased circ-Foxo3 levels (Supplementary Figure S1c). Cell lines 66C14, 4T1, MB-468, and MB-231 were transfected with this siRNA or a control oligo, followed by culturing in basal medium containing $1.2 \mathrm{mM}$ hydrogen peroxide for $24 \mathrm{~h}$. The

\footnotetext{
${ }^{1}$ Sunnybrook Research Institute, Sunnybrook Health Sciences Centre, Toronto, ON, Canada; ${ }^{2}$ Department of Laboratory Medicine and Pathobiology, University of Toronto, Toronto, ON, Canada; ${ }^{3}$ China-Japan Union Hospital of Jilin University, Jilin, China and ${ }^{4}$ Institute of Medical Science, University of Toronto, Toronto, ON, Canada ${ }^{*}$ Corresponding author: BB Yang, Sunnybrook Research Institute, University of Toronto, S-Wing Research Building, 2075 Bayview Ave, Toronto M4N 3M5, Canada. Tel: +1 416480 5874; Fax: +1 4164805737; E-mail: byang@sri.utoronto.ca

${ }^{5}$ These authors contributed equally to this work.

Received 28.6.16; revised 11.10.16; accepted 14.10.16; Edited by M Oren; published online 25.11.16
} 


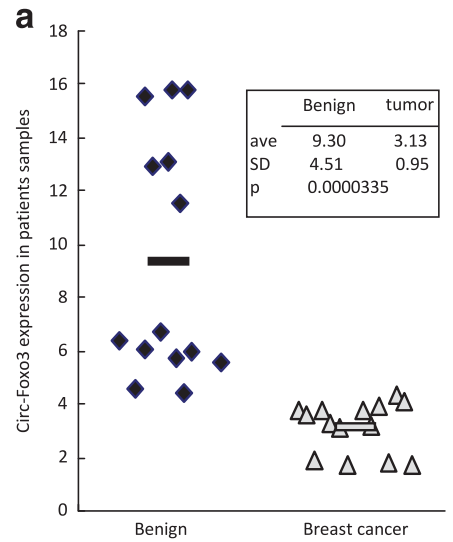

d

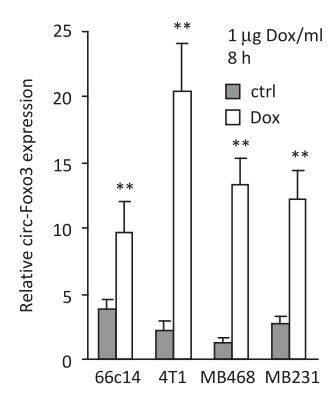

g

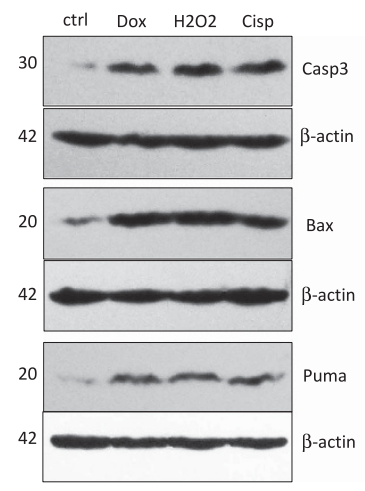

b
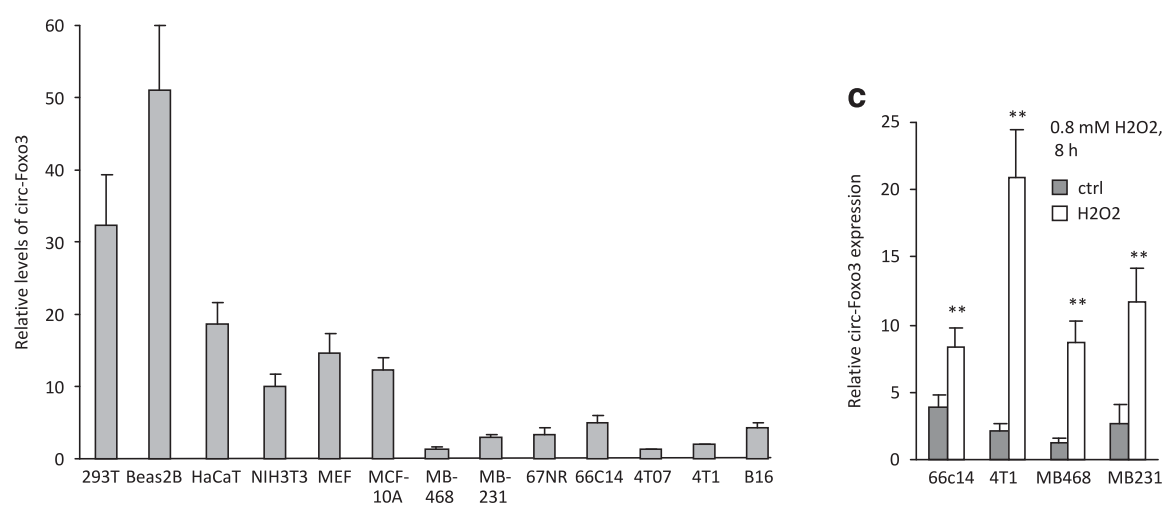

e

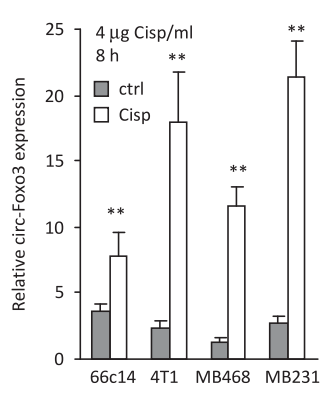

h

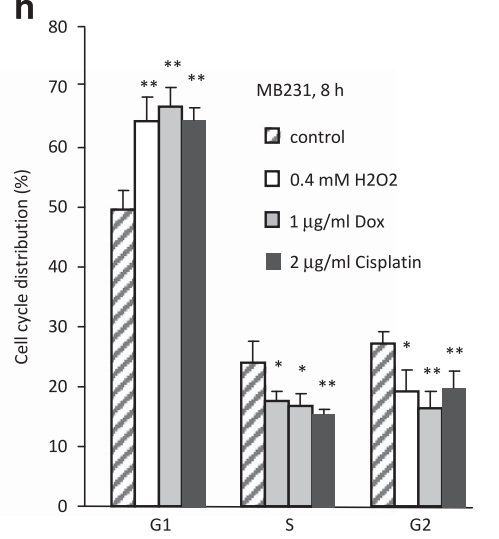

f

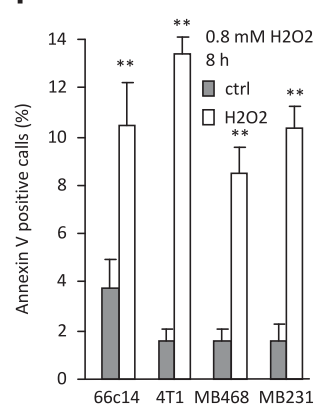

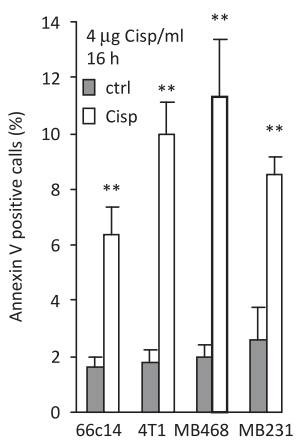

i
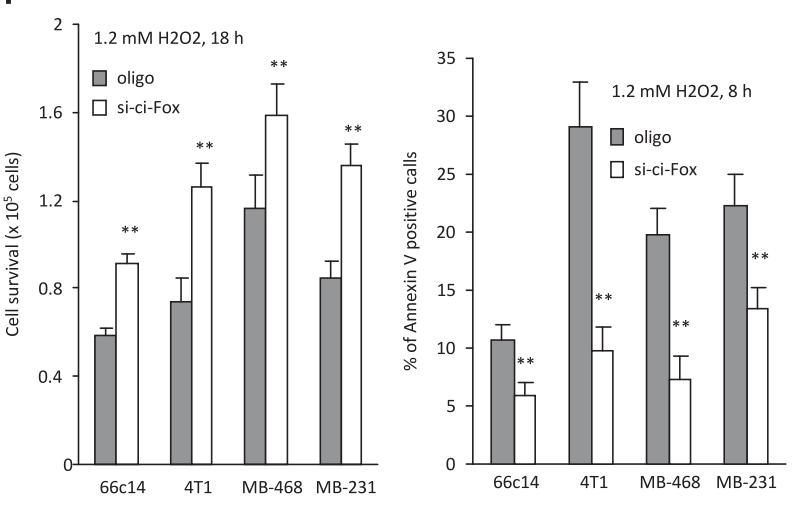

Figure 1 The effect of circ-Foxo3 on cell apoptosis. (a) Total RNAs were isolated from the specimens of patients with breast carcinoma and subject to real-time PCR measurement. Tumor samples showed significantly lower levels of circ-Foxo3 than the benign samples. (b) Expression of circ-Foxo3 was analyzed in a variety of cell lines by realtime PCR. Six non-cancer cell lines expressed significantly higher levels of circ-Foxo3 than seven cancer cell lines. (c-e) Cancer cell lines 66C14, 4T1, MDA-MB-468, and MDAMB-231 were cultured in the presence of $\mathrm{H} 2 \mathrm{O} 2$ (c), Dox (d), or Cisplatin (e). RNAs were isolated and subject to real-time PCR to measure circ-Foxo3 levels. Asterisks indicate significant differences. ${ }^{\star} P<0.05,{ }^{* *} P<0.01$. Error bars, S.D. $(n=3)$. (f) The cells were subject to Annexin-V staining to detect apoptosis. Treatment with $\mathrm{H} 2 \mathrm{O} 2$ (left), Dox (middle), or Cisplatin (right) increased apoptosis. (g) Western blot showed that $\mathrm{H} 2 \mathrm{O} 2$, Dox and Cisp treatment enhanced Bax and Puma expression and increased levels of cleaved Caspase-3 in MB-231 cells. (h) Cell cycle analysis showed that H2O2, Dox and Cisp treatment repressed cell cycle entry. ${ }^{*} P<0.05$, ${ }^{* *} P<0.01$. Error bars, S.D. ( $n=4$ ). (i) $66 \mathrm{C} 14,4 \mathrm{T1}$, MB-468, and MB-231 cells were transfected with circ-Fox03 siRNA or a control oligo. The cells were cultured in basal medium with $1.2 \mathrm{mM} \mathrm{H}_{2} \mathrm{O}_{2}$ for $18 \mathrm{~h}$ for survival assay (left) or maintained in $1.2 \mathrm{mM} \mathrm{H}_{2} \mathrm{O}_{2}$ for $8 \mathrm{~h}$ and subject to Annexin-V staining for apoptosis assay (right). Transfection with the circ-Foxo3 siRNA increased cell survival (left) and decreased apoptosis (right). ${ }^{\star} P<0.05,{ }^{\star \star} P<0.01$. Error bars, S.D. $(n=3)$

circ-Foxo3 siRNA-transfected cells showed enhanced cell viability (Figure 1i, left) but decreased apoptosis (Figure 1i, right, Supplementary Figure S1d), compared with controls. Similarly, the siRNA-transfected 4T1 cells showed enhanced cell viability (Supplementary Figure S2a, left) but decreased apoptosis (Supplementary Figure S2a, right, Supplementary Figure $\mathrm{S} 2 \mathrm{~b}$ ) after $\mathrm{H}_{2} \mathrm{O}_{2}$ treatment.
Ectopic circ-Foxo3 inhibited tumor growth and extended mouse lifespan. We further examined the role of circ-Foxo3 by stably transfecting $4 \mathrm{~T} 1$ cells with a circ-Foxo3 expression construct or a control vector. Expression of circ-Foxo3 was confirmed by RT-PCR (Supplementary Figure S2c), real-time PCR (Supplementary Figure S2d), and northern blotting (Supplementary Figure S2e). Ectopic circ-Foxo3 decreased 
cell survival in serum-free medium (Figure 2a) or when the cells were treated with $1.2 \mathrm{mM} \mathrm{H}_{2} \mathrm{O}_{2}$ for $16 \mathrm{~h}$ (Figure 2b) relative to the controls. We analyzed whether or not cell death occurred through apoptotic mechanisms. Three individual cell lines stably expressing circ-Foxo3 cultured in serum-free medium (Figure 2c, Supplementary Figure S2f) or treated with $0.6 \mathrm{mM} \mathrm{H}_{2} \mathrm{O}_{2}$ for $6 \mathrm{~h}$ (Figure 2d, Supplementary Figure S2g) were subject to Annexin-V staining. circ-Foxo3 expression was found to promote cell apoptosis.

The circ-Foxo3 construct was also stably expressed in cancer cell lines 66C14, 4T1, MB-468, and MB-231. The circ-Foxo3- and vector-transfected cells were cultured in serum-free medium for 6 days (Figure 2e) or treated with $1.0 \mathrm{mM} \mathrm{H}_{2} \mathrm{O}_{2}$ for $18 \mathrm{~h}$ (Supplementary Figure $\mathrm{S} 2 \mathrm{~h}$ ) to examine cell survival. Transfection with circ-Foxo3 decreased cell survival compared with the controls. By Annexin-V apoptosis assay, we detected increased apoptosis in cells transfected with circ-Foxo3 when the cells were cultured in serum-free medium (Figure 2f) or treated with $\mathrm{H}_{2} \mathrm{O}_{2}$ (Supplementary Figure S2i).

We then examined the effects of circ-Foxo3 in vivo. 4T1 cells transfected with circ-Foxo3 or a control vector were injected into regular Balb/c mice subcutaneously. We found that cells expressing circ-Foxo3 formed significantly smaller tumors than the control cells (Figure $2 g-h$ ). The experiment was repeated and similar results were obtained (Supplementary Figure S3a). The tumors were sectioned and subject to $\mathrm{H} \& \mathrm{E}$ and TUNEL staining. We detected extensive cell death in the tumors formed by cells transfected with circ-Foxo3, but not those formed by the control cells (Supplementary Figure S3b). A significant increased amount of TUNEL-positive cells were detected in tumors formed by the circ-Foxo3-transfected cells (Figure 2i). Analysis of the apoptosis-related genes revealed strong upregulation of Bax and Puma and increased levels of cleaved Caspase-3 in the tumors formed by the circ-Foxo3expressing cells (Figure 2j). Histological analysis revealed infiltration of B- and T cells into the circ-Foxo3 tumors and the surrounding connective tissues (Supplementary Figure S3c), suggesting an immune response of the hosts to the tumor xenografts.

circ-Foxo3- and vector-transfected MDA-MB-231 cells were also injected into nude mice. We found that the vector-transfected cells formed significantly larger tumors than the circ-Foxo3-transfected cells (Supplementary Figure S3d). H\&E staining of tumor sections showed extensive cell death in circ-Foxo3 tumors compared with the control (Supplementary Figure S3e). Apoptotic analysis indicated that the circ-Foxo3 tumors contained significantly greater number of TUNEL-positive cells than the control (Supplementary Figure S3f-h).

We explored the possibility that delivery of circ-Foxo3 plasmid could inhibit tumor growth in vivo. Four-week-old C57 mice were injected with wild type and circ-Foxo3 transfected B16 cells. The mice injected with wild type B16 cells were injected with a control vector or circ-Foxo3 plasmid conjugated with gold nanoparticles. This was repeated every 3 days until the experiment was completed on day 45. During this period, all 15 mice injected with B16 cells and control vector plasmid died, whereas 7 mice injected with the circ-Foxo3-transfected cells and 5 mice injected with B16 and the circ-Foxo3 plasmid independently survived (Supplementary Figure S4a). Statistical analysis showed significant effect of circ-Foxo3 on mouse survival (Figure 2k). Real-time PCR of the tumor tissues showed significantly higher levels of circ-Foxo3 in the mice injected with the circ-Foxo3 plasmids or the circ-Foxo3transfected cells (Figure 2l). We detected increased amounts of TUNEL-positive cells in the tumors from mice injected with circ-Foxo3 plasmids or the circ-Foxo3transfected cells (Figure $2 \mathrm{~m}$ ). The TUNEL-positive cells were mainly colocalized with the circ-Foxo3-positive cells (Supplementary Figure S4b), further confirming the effect of circ-Foxo3 on cancer cell apoptosis. We measured the levels of circ-Foxo3 in the transfected cells and non-cancer cells and found that the levels were comparable (Figure 2n), suggesting that the levels of circ-Foxo3 overexpression was physiologically relevant.

circ-Foxo3 affects expression of Foxo3, p53, and Puma. We tested the potential interaction of circ-Foxo3 with proteins associated with apoptosis. Cell lysis prepared from MB-231 cells were subject to immunoprecipitation with antibodies against MDM2, p53, Puma, and Foxo3, followed by real-time PCR with primers specific for linear Foxo3 or circ-Foxo3. circ-Foxo3 was pulled down by antibodies against MDM2 and p53, which did not pull-down linear Foxo3 mRNA (Figure 3a). RNAs prepared from the circ-Foxo3- and vector-transfected cells were subject to pull-down assay. Antibodies against MDM2 and p53 were able to pull-down significantly higher levels of circ-Foxo3 from the circ-Foxo3 cells, than those from the control cells (Figure 3b).

We also prepared lysates from another cell line BEAS2B, which expressed extremely high levels of circ-Foxo3, and performed the pull-down assay using the biotinylated probe with different doses. Real-time PCR showed that pulling-down circ-Foxo3 was dependent on the doses of the probe, reaching a plateau at the dose of $10^{-2} \mu \mathrm{g}$ (Figure 3c). After incubation and pull-down by the probe, the remaining lysates were subject to western blot analysis for levels of MDM2 and p53. Both MDM2 and p53 showed decreased levels after circ-Foxo3 was pulled down when the circ-Foxo3 probe was at the doses over $10^{-2} \mu \mathrm{g}$ (Figure $3 \mathrm{~d}$ ).

We also analyzed the expression of MDM2, p53, and Puma. Ectopic expression of circ-Foxo3 had no effect on p53 mRNA levels (Figure $3 e$ ) but decreased p53 protein, increased Foxo3 and Puma levels (Figure 3f). The cells were also treated with the proteasome inhibitor MG-132, followed by western blotting. The levels of p53, Foxo3, and Puma appeared similar in the cells treated with MG-132 (Figure $3 \mathrm{~g}$ ), suggesting that the changes in protein levels were a consequence of protein degradation. To test if the binding interaction was circular RNA specific, we measured the levels of other randomly selected circular RNAs. Similar to the negative controls, antibodies against MDM2 and p53 did not pull-down these circular RNAs (Figure 3h).

To validate that the changes in p53, Foxo3, and Puma were associated with protein degradation owing to circ-Foxo3, we treated cells with $20 \mu \mathrm{M}$ Emetine, an agent that blocks protein synthesis. The levels of p53 in the circ-Foxo3-transfected cells decreased at a higher rate than in the control cells after long-term treatment (Figure 3i, upper). Three independent 
experiments were performed and the proteins bands were quantified by densitometry. A significant difference was observed following $1 \mathrm{~h}$ treatment with Emetine (Supplementary Figure S5a, left). Treatment with Emetine decreased Foxo3 levels in the control cells at a higher rate than in the circ-Foxo3-transfected cells (Figure 3i, lower), reaching significant difference when the cells were treated for $8 \mathrm{~h}$ (Supplementary Figure S5a, right). We then validated the effects of circ-Foxo3 in regulating levels of p53 and Foxo3 using siRNAs. MB-231 cells transfected with siRNA-targeting
circ-Foxo3 were confirmed to show increased p53 levels and decreased Foxo3 levels (Figure 3j, Supplementary Figure S5b). We also examined expression of Foxo3, p53, Mdm2, and Puma in tumor sections from 4T1 (Supplementary Figure S5c) and MDA-MB-231 xenografts (Supplementary Figure S5d). Although we did not detect a clear difference in Mdm2 levels, we detected increased expression of Foxo3 and Puma, but decreased levels of p53 in the circ-Foxo3 tumor sections, which were consistent with the apoptosis data previously obtained.

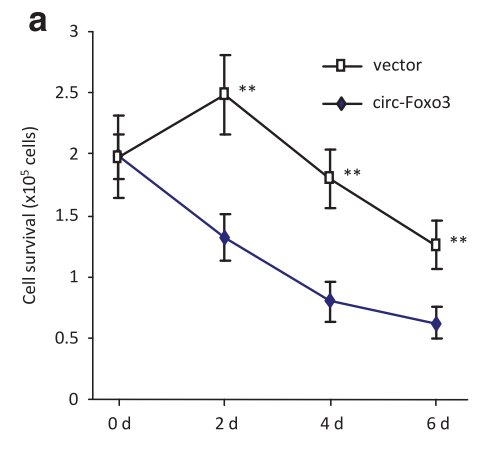

e

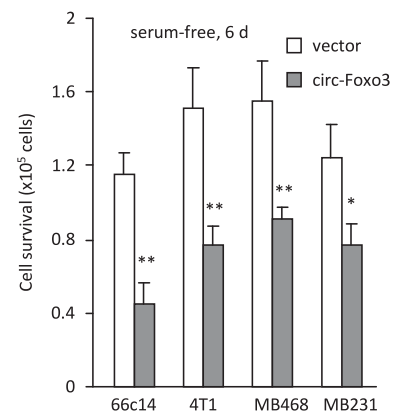

h

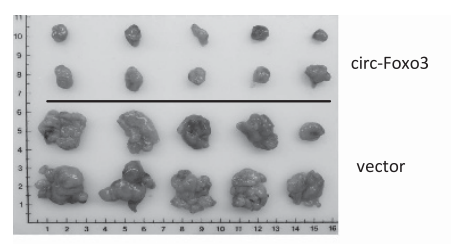

$\mathbf{k}$

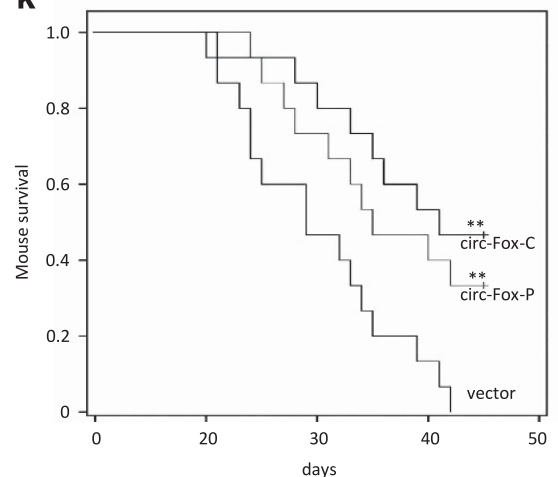

b

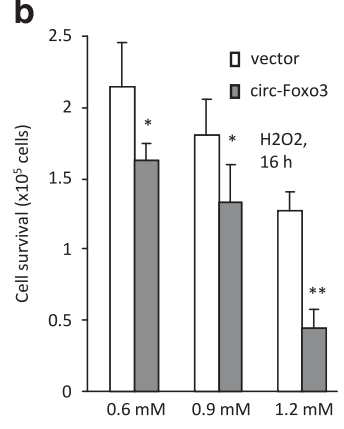

c

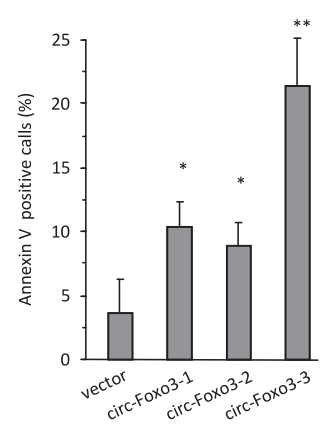

d

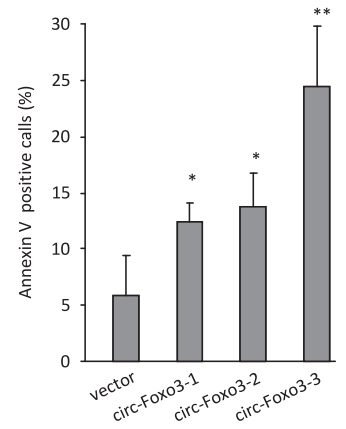

f

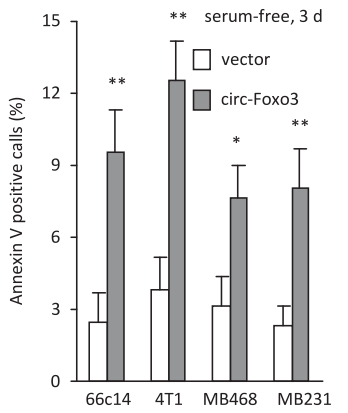

9

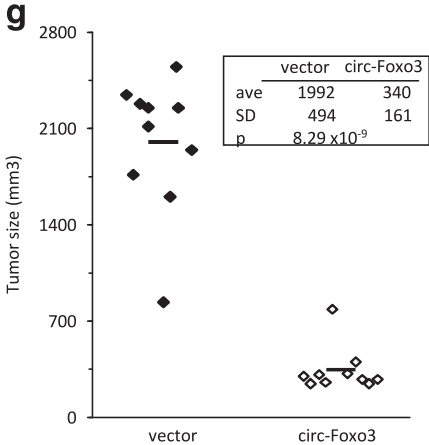

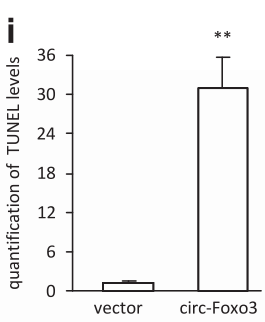

I

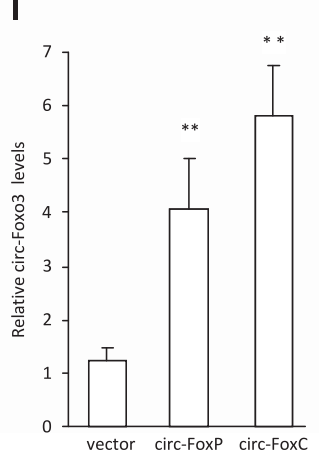

j
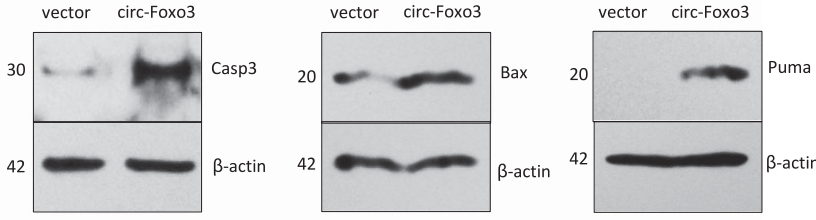

m

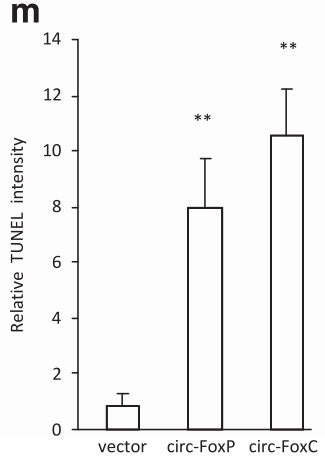

n

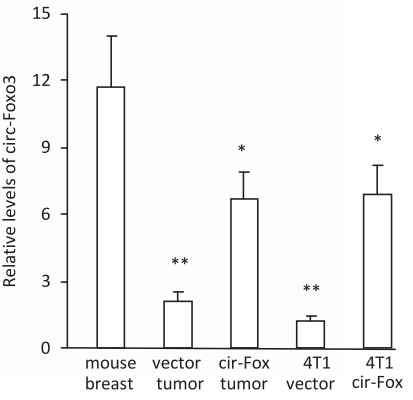


Interaction of circ-Foxo3 with MDM2 and p53. Western blot analysis indicated that altthough the levels of MDM2 were not affected, p53 levels decreased, but expression of Foxo3 and Puma increased in the circ-Foxo3 tumors relative to the control (Figure 4a). In situ hybridization displayed colocalization of circ-Foxo3 with Mdm2, p53, Foxo3, and Puma (Figure 4b, full panels in Supplementary Figure S6 and Supplementary Figure S7). Quantification analysis showed that levels of circ-Foxo3 were significantly higher in the tumor tissues obtained from the mice injected with circ-Foxo3 plasmids or the circ-Foxo3-transfected cells (Figure 4c). Levels of p53 but not MDM2 decreased. As well, Foxo3 and Puma levels increased in the circ-Foxo3 groups (Figure 4d).

We explored the possibility that circ-Foxo3 interacted with MDM2 and p53. Identification of secondary structure is the first step toward determining and studying the RNA tertiary conformation. In the secondary structures of circular RNAs, the first and last base remains adjacent in the ring molecule and they do not pair with each other. ${ }^{17}$ To determine the secondary structures with lowest free energy, we used minimum free energy algorithm implemented in Mfold (version 2.3). ${ }^{18}$ Mfold is a dynamic programming algorithm, which uses energy minimization to model the ensemble of possible structures by identifying optimal folding of a nucleic-acid sequence within a specified energy increment. Thus, the secondary structure with a lower theoretical value of free energy was selected as a model structure for 3D structure prediction. The output file containing primary sequence and an associated secondary structure (Dot-Bracket Notation) was then submitted to RNA Composer ${ }^{19}$ to generate the $3 D$ structure. RNA composer is based on the machine translation principle and is a web-based RNA folding application used to assemble a 3D RNA structure.

The best predicted secondary structure of circ-Foxo3 was derived by analyzing its thermodynamic properties using the formula $\Delta \mathrm{G}=\Delta \mathrm{H}-\mathrm{T} \Delta \mathrm{S}, \Delta \mathrm{G}=-426.70 \mathrm{kcal} / \mathrm{mol}$ at $37^{\circ} \mathrm{C}$, $\Delta \mathrm{H}=-4417.50 \mathrm{kcal} / \mathrm{mol}$, and $\Delta \mathrm{S}=-12867.3 \mathrm{cal} /(\mathrm{K} \cdot \mathrm{mol})$, where $\mathrm{T}(\mathrm{K})$ is the absolute temperature and $\Delta \mathrm{G}, \Delta \mathrm{H}, \Delta \mathrm{S}$, denote the change in free energy, enthalpy, and entropy, respectively. The secondary structure defined using dotbracket notation was then analyzed by the software RNA composer for tertiary structure prediction. NPDock was then used to carry out the in-silico molecular docking between circFoxo3 and MDM2 (RING-finger domain) (Figure 4e). The residues 429-491 in the MDM2 RING-finger domain used in the docking procedure were derived from Protein Data Bank entry 2HDP. The molecular simulation result supported that circ-Foxo3 could perfectly dock MDM2 and predicted a minimal binding region of circ-Foxo3 for the MDM2 as 'agu cgauucaugcggguccagaaugagggaacuggcaaga' (Supplementary Figure S8a).

As p53 interacts with RNA via its C-terminal regulatory domain, ${ }^{13}$ we used this domain in the docking analysis. The p53 (C-terminal regulatory domain) used in the docking procedure was derived from Protein Data Bank entry 1DT7. Using similar approaches, we confirmed that circ-Foxo3 docked p53 C-terminal regulatory domain (Figure 4f). The molecular simulation result supported that circ-Foxo3 could perfectly dock p53 and predicts a minimal binding region of circ-Foxo3 for p53 as 'gggugccaggcugaaggaucacug' (Supplementary Figure S8a).

The contact distance between circ-Foxo3 and MDM2 binding domain (Supplementary Figure S8b), the MC score (Supplementary Figure $4 \mathrm{~g}$ ), and the residue-level resolution contact maps (Supplementary Figure S8c) all supported the conclusion that circ-Foxo3 sufficiently docked MDM2. The contact distance between circ-Foxo3 and p53-binding domain (Supplementary Figure S8d), the MC score (Figure $4 \mathrm{~h}$ ), and the residue-level resolution contact maps (Supplementary Figure S8e) all supported the conclusion that circ-Foxo3 sufficiently docked p53 C-terminal regulatory domain.

To validate the interaction, we transfected siRNA-targeting circ-Foxo3 into MB-231 cells and performed a pull-down experiment. After we confirmed that the siRNA knocked down circ-Foxo3 (Supplementary Figure S9a), we found that the biotinylated probe pulled down significantly lower levels of circFoxo3 when the cells were transfected with the siRNA (Figure 5a). In the protein pull-down assay, we confirmed that p53 expression increased in the lysate prepared from circFoxo3-siRNA-transfected cells, when compared with the vector-transfected cells, but there was an equal amount of MDM2 in both (Figure 5b, upper). Even with the increased level of p53, we found that the biotinylated probe pulled down

\footnotetext{
Figure 2 Expression of circ-Fox03 inhibited tumor growth and enhanced mouse survival. (a) Cultured in serum-free medium for 6 days, the circ-Foxo3-transfected 4T1 cells showed decreased cell survival compared with mock control cells. ${ }^{*} P<0.05,{ }^{*} P<0.01$. Error bars, S.D. $(n=6)$. (b) The transfected $4 \mathrm{~T} 1$ cells were cultured in basal medium with $\mathrm{H}_{2} \mathrm{O}_{2}$ for $16 \mathrm{~h}$ for survival assay. ${ }^{*} P<0.05,{ }^{* *} P<0.01$. Error bars, S.D. $(n=6)$. (c) The transfected $4 \mathrm{~T} 1$ cells were cultured in serum-free medium for $48 \mathrm{~h}$, and subjected to Annexin-V staining, followed by flow cytometry to measure apoptotic cells. ${ }^{*} P<0.05,{ }^{* \star} P<0.01$. Error bars, S.D. $(n=4)$. (d) The cells were cultured in basal medium with 0.6 mM $\mathrm{H}_{2} \mathrm{O}_{2}$ for $6 \mathrm{~h}$, and subjected to Annexin-V staining, followed by flow cytometry to measure apoptotic cells. ${ }^{*} P<0.05,{ }^{* *} P<0.01$. Error bars, S.D. ( $n=4$ ). (e) The circ-Foxo3- and mock-transfected cells 66C14, 4T1, MDA-MB-468, and MDA-MB-231 were cultured in serum-free medium for 6 days for cell survival assay. The circ-Foxo3-transfected cell lines showed decreased viability compared with the control. ${ }^{*} P<0.05$, ${ }^{*} P<0.01$. Error bars, S.D. $(n=3)$. (f) The cells were cultured in serum-free medium for 3 days, followed by apoptotic assay. circ-Fox03 expression enhanced apoptosis. ${ }^{*} P<0.05,{ }^{*} P<0.01$. Error bars, S.D. $(n=3)$. (g-h) $4 T 1$ cells transfected with circ-Fox03 and the vector were subject to tumorigenesis assay. Expression of circ-Foxo3 inhibited tumor growth (g). Typical photo of tumors (h). (i) Tumor sections were subject to TUNEL staining followed by quantification of the TUNEL-positive cells. Significantly higher levels of cell death were detected in the tumors formed by cells transfected with circ-Foxo3 compared with the control. (j) Western blot showed that the tumor tissues expressing high levels of circ-Foxo3 had increased levels of Bax, Puma, and cleaved Caspase-3. (k) Mice were intraperitoneally injected with B16 cells with a control vector or circ-Fox03 plasmid, and with circ-Fox03 transfected B16 cells, followed by survival test. Kaplan-Meier curve was obtained. Injection of circ-Fox03 plasmid or circ-Fox03-transfected B16 cells increased mouse survival significantly $\left({ }^{* *} P<0.01\right)$. (I) PCR showed that circ-Fox3 expressed at high levels in plasmid injection and circ-Foxo3-transfected groups of tumors. $(\mathrm{m})$ In TUNEL staining, significantly higher levels of cell death were detected in the tumors of mice injected with circ-Fox03 plasmids or circ-Fox03-transfected cells relative to the control. (n) PCR showed that normal mouse breast tissues expressed high levels of circ-Foxo3 compared with $4 \mathrm{~T} 1$ tumors and cell lines. ${ }^{*} P<0.05,{ }^{* *} P<0.01$. Error bars, S.D. $(n=4)$
} 


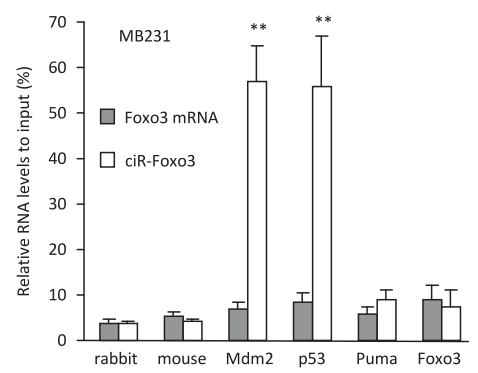

b

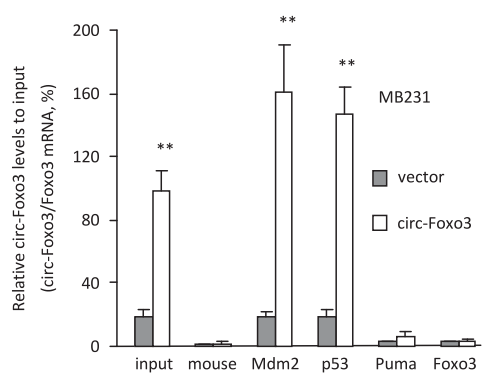

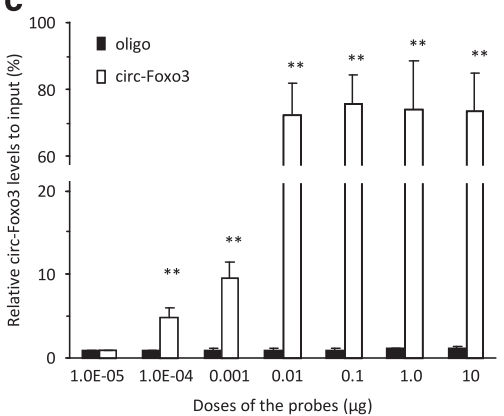

d
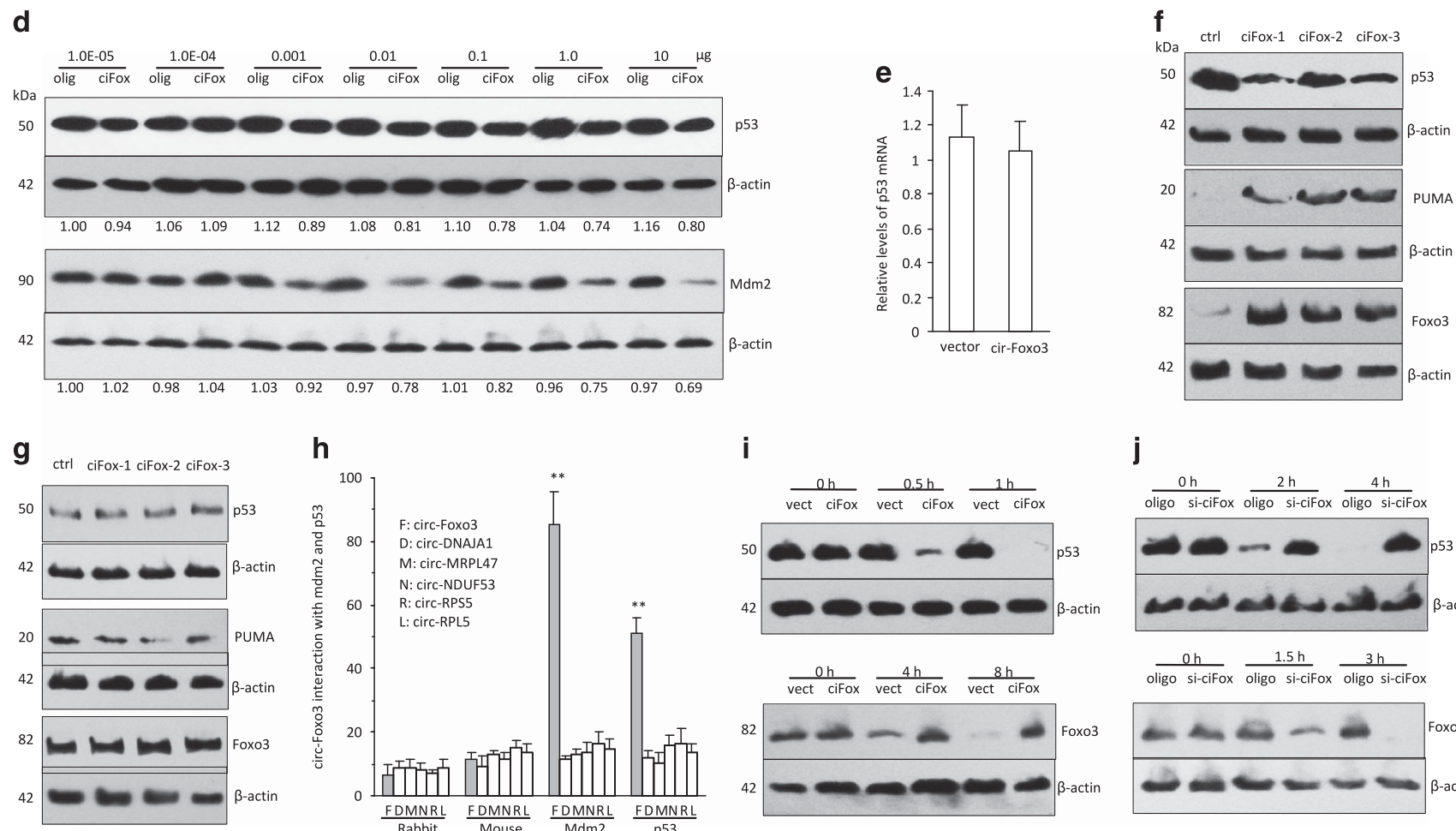

h

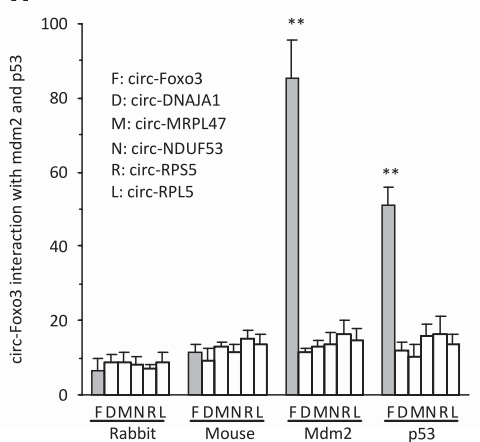

i
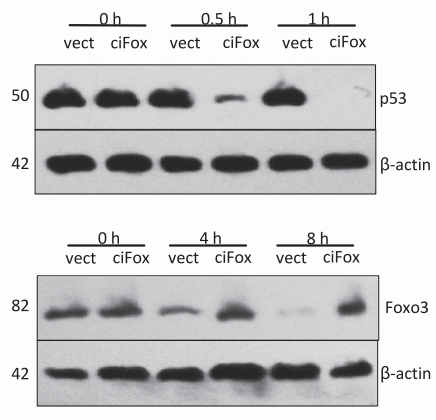

j

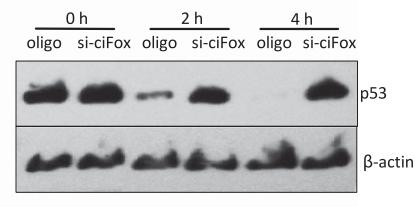

$\frac{0 \mathrm{~h}}{\text { oligo si-ciFox }} \frac{1.5 \mathrm{~h}}{\text { oligo si-ciFox }} \frac{3 \mathrm{~h}}{\text { oligo si-ciFox }}$

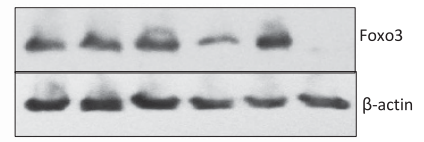

Figure 3 Expression of circ-Foxo3 decreased p53 but increased Foxo3 levels. (a) MB-231 cell lysis were subject to immunoprecipitation with anti-rabbit lgG, mouse lgG, MDM2, p53, Puma, and Fox03 antibodies, followed by real-time PCR with primers specific for Fox03 or circ-Foxo3. Antibodies against MDM2 and p53 pulled down circ-Foxo3, but not Fox03 mRNA. ${ }^{* \star} P<0.01$. Error bars, S.D. $(n=4)$. (b) Cell lysates prepared from MB-231 cells transfected with circ-Foxo3 or the mock control were subject to immunoprecipitation with different antibodies, followed by real-time PCR. Antibodies against MDM2 and p53 pulled down more circ-Fox03 from the circ-Fox03-transfected cells than from the control cells. ${ }^{* *} P<0.01$. Error bars, S.D. $(n=4)$. (c) Lysates prepared from BEAS2B cells were hybridized with biotinylated probe with increased concentration, and subject to RNA pull-down assays. Real-time PCR showed that circ-Foxo3 pulled down high levels of circ-Foxo3, reaching plateau at the dose of $10^{-2} \mu \mathrm{g} .{ }^{* *} P<0.01$. Error bars, S.D. $(n=4)$. (d) After being pulled down by the circ-Foxo3 probe, the remaining lysates were subject to western blotting. Both MDM2 and p53 showed decreased levels after circFox03 was pulled down with the circ-Fox03 probe at the doses over $10^{-2} \mu \mathrm{g}$. (e) PCR showed that overexpression of circ-Fox03 did not change p53 mRNA levels. (f) Lysates prepared from MB-231 cells transfected with mock control or circ-Fox03, were subject to western blot analysis. Expression of circ-Foxo3 decreased p53 levels, but increased

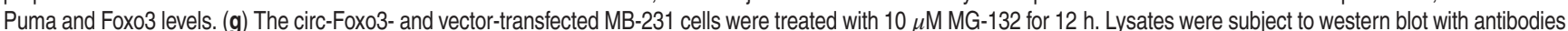
against p53, Puma, and Fox03. Little change was detected. (h) The pulled down pellets were also measured for the levels of other circular RNAs including circ-DNSJA1, circMRPL47, circ-NDUF53, circ-RPS5, and circ-PRL5. Antibodies against MDM2 and p53 could only pull-down circ-Foxo3. (i) The circ-Foxo3- and vector-transfected MB-231 cells were cultured in basal medium containing $20 \mu \mathrm{M}$ Emetine for the time points as indicated, followed by western blotting. circ-Foxo3 expression decreased p53 (upper) but increased Foxo3 (lower) levels compared with mock control. (j) Lysates prepared from MB-231 cells transfected with circ-Foxo3 siRNA or a control oligo were treated with $20 \mu \mathrm{M}$ Emetine for the time points as indicated, followed by western blotting. Transfection with circ-Fox03 siRNA increased p53 (upper) but decreased (Fox03) levels (lower)

lower levels of p53 and MDM2 in the siRNA-transfected cells than those in the control oligo group (Figure $5 b$, lower). The tumors formed by the circ-Foxo3-transfected cells showed increased levels of Foxo3 and Puma, but decreased levels of p53 compared with the controls (Figure $5 \mathrm{c}$ ). There was little difference in the levels of MDM2.
We further confirmed that circ-Foxo3 mediated the formation of p53-MDM2 complex by testing whether p53-MDM2 co-IP was altered by circ-Foxo3 mRNA overexpression or knockdown and by RNAse-A treatment. We performed experiments using MDM2 antibody to pull-down p53 with or without circ-Foxo3 siRNA and with or without RNAse-A 
a

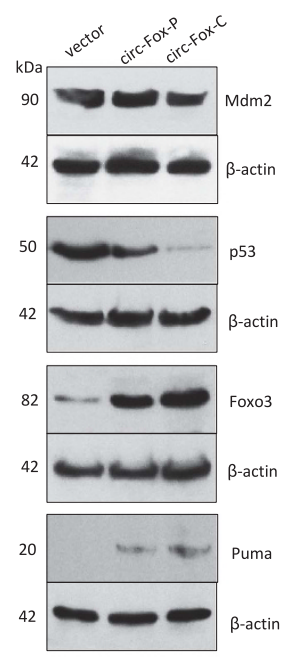

C

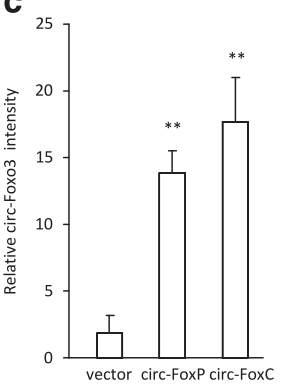

d

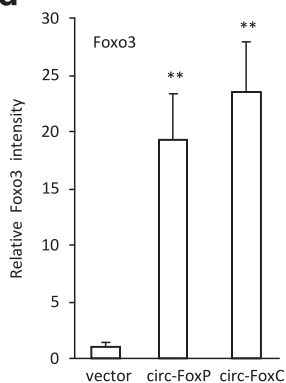

b
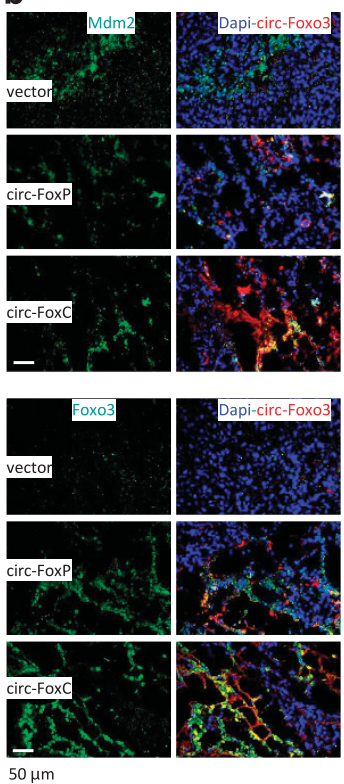
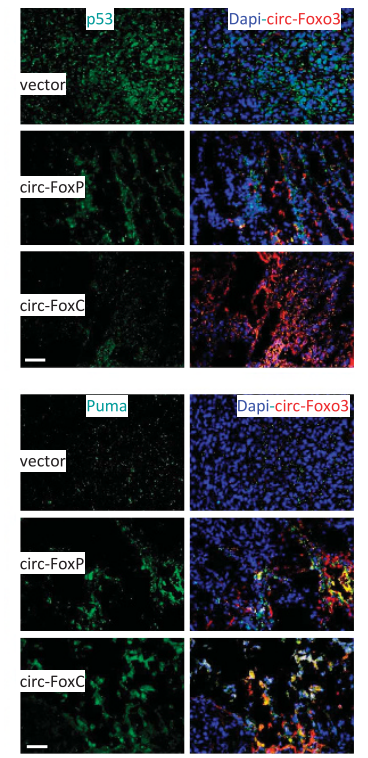
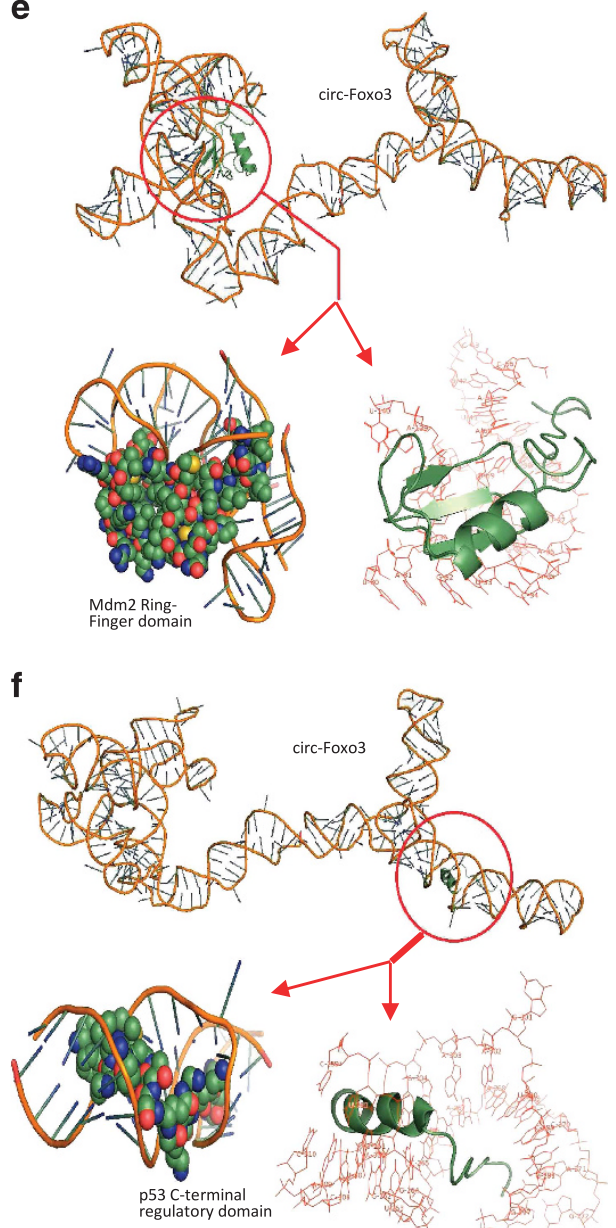

h

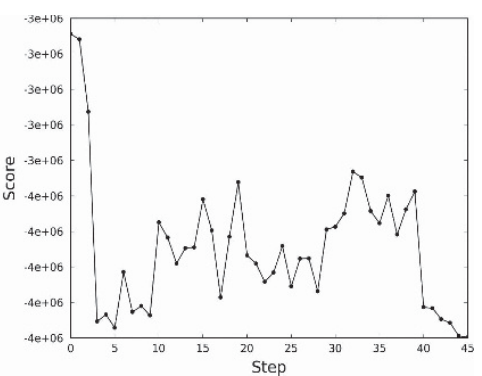

Figure 4 Interaction of circ-Foxo3 with MDM2 and p53 (a) B16 tumor lysates were subject to western blot with antibodies to p53, Foxo3, Puma, Mdm2 and $\beta$-actin. circ-Foxo3 enhanced Fox03 and Puma expression and repressed p53 expression in the tumors. (b) Tumor sections were stained with DAPI (blue), circ-Foxo3 (red, by in situ hybridization) and related proteins including Mdm2, p53, Foxo3, and Puma (green, by immunohistochemical staining). Typical photos showed that expression of circ-Foxo3 was colocalized with these protein. (c) Quantification of circ-Foxo3, Mdm2, and p53 in the tumor sections. (d) Quantification of Foxo3 and Puma in the tumor sections. (e) Graphical representation of three-dimensional structures of circ-Foxo3 and MDM2 (RING-finger domain) docking models with a zoom-in image of the binding interface done by NPDock. The binding region is shown in two different visualizations (cartoon and sphere). (f) Graphical representation of three-dimensional structures of circ-Foxo3 and p53 (C-terminal regulatory domain) docking models with a zoom-in image of the binding interface done by NPDock. The binding region is shown in two different visualizations (cartoon and sphere). (g) Refinement of the best docked circ-Foxo3-MDM2 (RING-finger domain) model showing MC score vs steps of simulation. (h) Refinement of the best docked circ-Foxo3-p53 (C-terminal regulatory domain) model showing $\mathrm{MC}$ score vs steps of simulation. ${ }^{\star} P<0.05,{ }^{\star \star} P<0.01$

treatments. We also pulled down MDM2 using p53 antibody with or without circ-Foxo3 siRNA and with or without RNAse-A treatment. Our experiments confirmed the essential role of circ-Foxo3 in the formation of p53-MDM2 complex (Figure 5d).
We identified the binding sites and generated mutations as shown (Figure 6a) by using primers listed (Supplementary Figure S9b). Real-time PCR analysis indicated that transfection with neither the mutation construct nor the blocking oligos affected levels of circ-Foxo3 products (Figure 6b). However, 
antibody against p53 could not pull-down the mutants harboring mutation in the p53-binding site or when the cells were co-transfected with circ-Foxo3 and blocking oligo for p53binding site (Figure 6c). Similarly, antibody against Mdm2 could not pull-down the mutants harboring mutation in the Mdm2-binding site or when the cells were co-transfected with circ-Foxo3 and blocking oligo for Mdm2-binding site. The circFoxo3 probe could precipitate MDM2 and p53 in the circFoxo3 expressing cells, but not in the cells transfected with the respective mutants (Figure $6 \mathrm{~d}$ ) or the blocking oligos (Figure 6e). Precipitation of Mdm2 pulled down p53 and precipitation of p53 pulled down Mdm2, in the circ-Foxo3transfected cells, but not in the binding site mutated cells or in the cells co-transfected with the blocking oligos (Figure 6f). The effects of circ-Foxo3 on cell survival (Figure 6g) and apoptosis (Figure 6h) were abolished when the cells were transfected with the mutation constructs or the blocking oligos.

We validated the role of Mdm2 in mediating circ-Foxo3's effects by designing three siRNAs targeting Mdm2 (Figure 7a). Western blotting showed that silencing Mdm2 enhanced expression of p53, Foxo3 and Puma (Figure 7c). Silencing MDM2 enhanced Foxo3 expression when the cells were treated with Emetine (Figure 7b). Silencing MDM2 decreased cell survival and increased cell apoptosis (Figure 7d).

We examined the effect of p53 in mediating the function of circ-Foxo3. western blot analysis showed that transfection with circ-Foxo3 enhanced Foxo3 and Puma expression when the cells were transfected with p53 siRNA or p53 expression construct (Figure 7e), suggesting that p53 had little effect on circ-Foxo3 upregulation of Foxo3 and Puma. Functionally, transfection with circ-Foxo3 repressed cell survival and increased cell apoptosis when the cells were transfected with
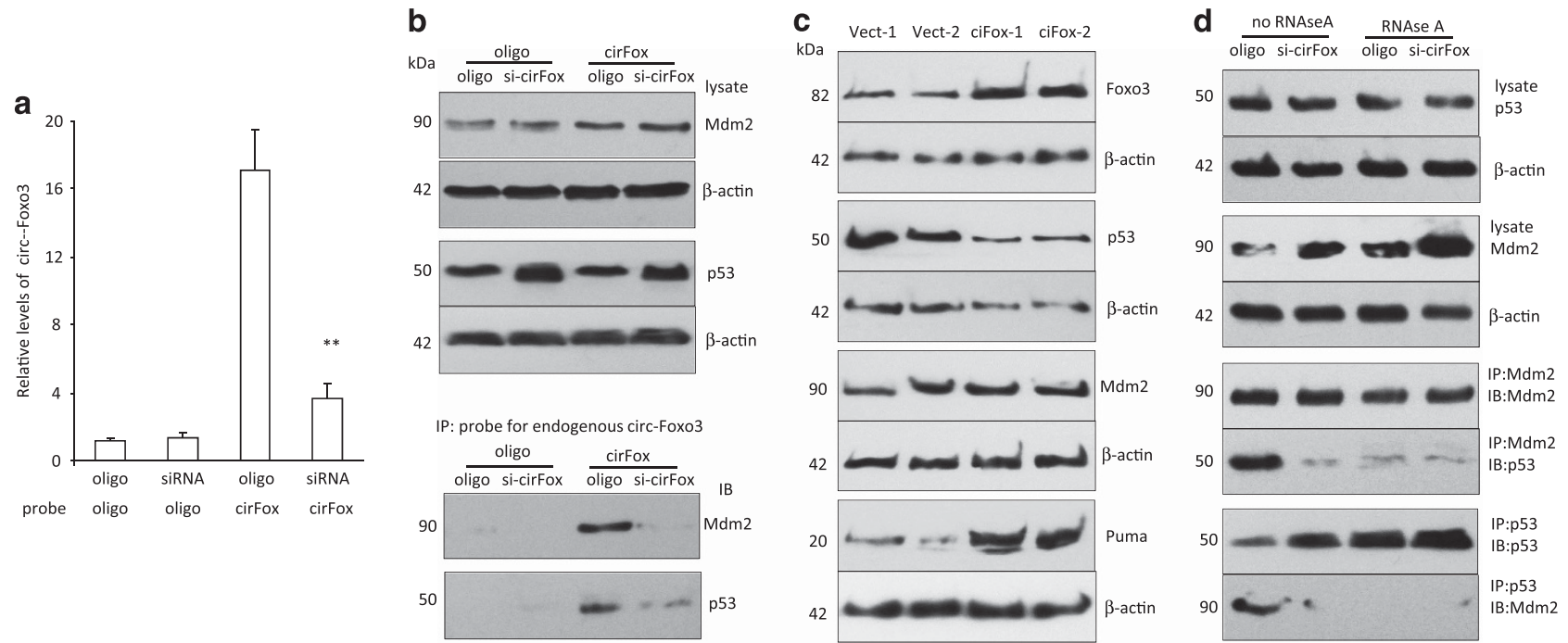

Figure 5 The interaction of circ-Fox03 with p53 and MDM2. (a) Lysates prepared from MB-231 cells transfected with circ-Fox03 siRNA or a control oligo were hybridized with the biotinylated probe. Real-time PCR showed that circ-Foxo3 probe pulled down less circ-Foxo3 in the circ-Foxo3 siRNA-transfected cells. ${ }^{* *} P<0.01$. Error bars, S.D. $(n=4)$. (b) Upper, the lysates were subject to western blot with antibodies against MDM2 and p53. Increased p53 levels were detected. Lower, the lysates were subject to RNA pull-down assays. The circ-Fox03 probe pulled down less MDM2 and p53 in the cells transfected with the circ-Fox03 siRNA. (c) The tumors formed by the circ-Foxo3-transfected cells revealed higher levels of Fox03 and Puma, but lower levels of p53 compared with the controls. There was little difference in the levels of MDM2. (d) Lysates prepared from MB-231 cells transfected with circ-Foxo3 siRNA or a control oligo were incubated with or without $1 \mu \mathrm{g} / \mathrm{ml}$ RNAse-A for $30 \mathrm{~min}$. Antibody against MDM2 pulled down decreased levels of p53 and antibody against p53 pulled down decreased levels of MDM2, when circ-Foxo3 was silenced or treated with RNAse-A

Figure 6 Mutation of circ-Fox03 in the MDM2 and p53-binding sites. (a) Sequences of circ-Foxo3 with Mdm2 and p53-binding sites, designed mutated binding sites, and blocking oligos. (b) PCR showed the expression of circ-Foxo3 in cell lysates prepared from MB-231 cells transfected with the control vector, oligo, circ-Foxo3, circ-Fox03 mutated in p53-binding sites (mut-p53), circ-Foxo3 mutated in Mdm2-binding sites (mut-mdm2), circ-Foxo3 mutated in both p53 and Mdm2-binding sites (mut-B), circ-Foxo3 and p53 blocking oligo (block-p53), circ-Fox03 and Mdm2 blocking oligo (block-mdm2), and circ-Foxo3 and both p53 and Mdm2 blocking oligos (block-B). (c) Real-time PCR showed that antibodies against MDM2 and p53 pulled down more circ-Foxo3 from the circ-Foxo3-transfected cells than from the control cells, but not in the binding site mutated cells or cells treated with blocking oligos. ${ }^{* *} P<0.01$. Error bars, S.D. $(n=4)$. (d) Cell lysates prepared from MB-231 cells transfected with the control vector (vect), oligo, circ-Foxo3, mutated p53 (mup53), mutated Mdm2 (muMd), mutated in both p53 and Mdm2-binding sites (mu-B) were subject to circ-Fox03 pull-down assays. The circ-Fox03 probe pulled down MDM2 and p53 of circ-Fox03 expressing cells, but not the binding site mutated cell lines. (e) Cell lysates prepared from MB-231 cells transfected with the vector, oligo, circ-Fox03, circ-Fox03 and blop53, circ-Fox03 and bloMdm2, and circ-Fox03 and block-B. The circ-Foxo3 probe pulled down MDM2 and p53 in circ-Foxo3 expressing cells, which was blocked by the blocking oligos. (f) Western blot showed p53 and Mdm2 expression in MB-231 cells transfected with different constructs. Precipitation of Mdm2 pulled down p53 and precipitation of p53 pulled down Mdm2, in the circ-Fox03 overexpressing cells, but not in the binding site mutated cells nor cells co-transfected with the blocking oligos. (g) The above cells were cultured in $0.8 \mathrm{mM} \mathrm{H} 2 \mathrm{O} 2$ for $24 \mathrm{~h}$. Expression of circ-Foxo3 repressed cell survival, which could be prevented by binding site mutation or application of the blocking oligos. (h) The cells were cultured in $0.8 \mathrm{mM} \mathrm{H} 2 \mathrm{O} 2$ for $10 \mathrm{~h}$. Expression of circ-Foxo3 enhanced cell apoptosis, which could be prevented by binding site mutation or application of the blocking oligos. ${ }^{*} P<0.05,{ }^{* *} P<0.01$. Error bars, S.D. $(n=3)$ 
p53 siRNA or p53 expression construct (Figure 7f), suggesting that p53 had little effect on circ-Foxo3 mediating cell survival and apoptosis.
circ-Foxo3 modulated the function of MDM2 in ubiquitinating p53 and Foxo3. We further examined how circFoxo3 modulated MDM2 by decreasing p53 and increasing
circ-Foxo3, Mdm2 binding site in $5^{\prime}$ underlined,

(

5'aacuccauccggcacaaccugucacugcauagucgauucaugcggguccagaaugaggg aacuggcaagagcucuugguggaucauca... ggaacuucacuggugcuaagcaggccuca cucagagcugggugccaggcugaaggaucacugaggaaggggaagugggcaaagcag $3^{\prime}$

circ-Foxo3, mutated Mdm2 binding site

5'aacuccauccggcacaaccugucacugcauucagcuaaguccgaaagggucucccuccc ungaggcaagagcucuugguggaucauca...ggaacuucacuggugcuaagcaggccuca cucagagcugggugccaggcugaaggaucacugaggaaggggaagugggcaaagcag $3^{\prime}$

circ-Foxo3, mutated p53 binding site

5 'aacuccauccggcacaaccugucacugcauagucgauucaugcggguccagaaugaggga acuggcaagagcucuugguggaucauca...ggaacuucacuggugcuaagcaggccucauc ucagagcuuuuacgguccgacuuccuagugacaggaaggggaagugggcaaagcag $3^{\prime}$

Mdm2 blocking oligo $5^{\prime}$ ctcttgccagttccctcattctggacccgcatgaatcgacta p53 blocking oligo 5' cctcagtgatccttcagcctggcacccagc

control oligo $5^{\prime}$ tccttcctctctttctctcccttgtga

b

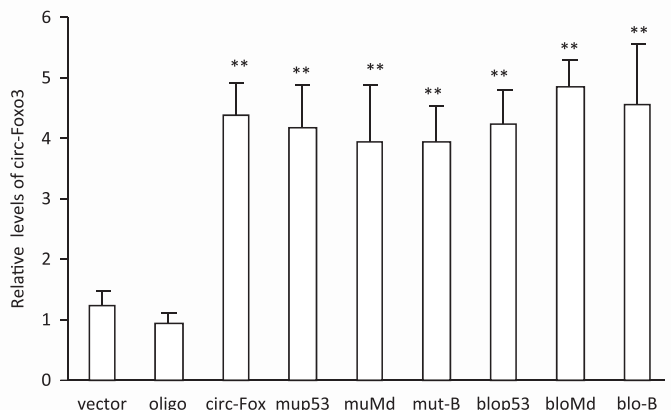

e
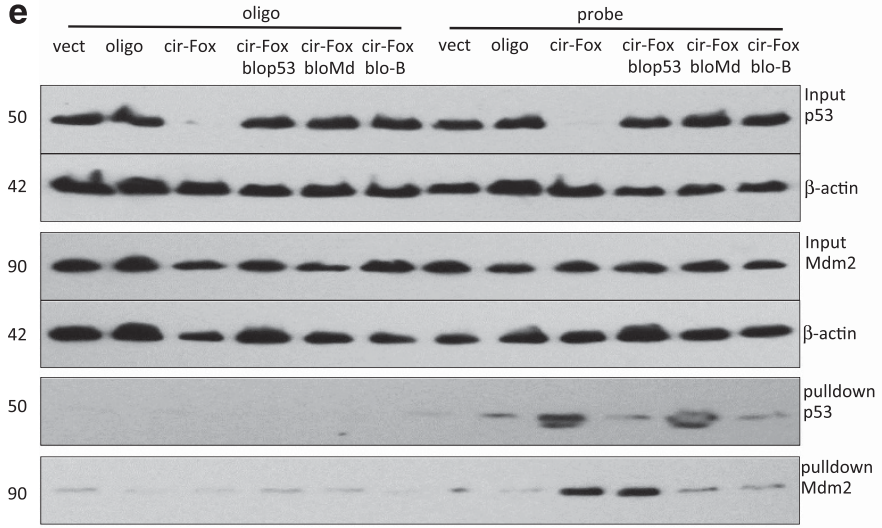
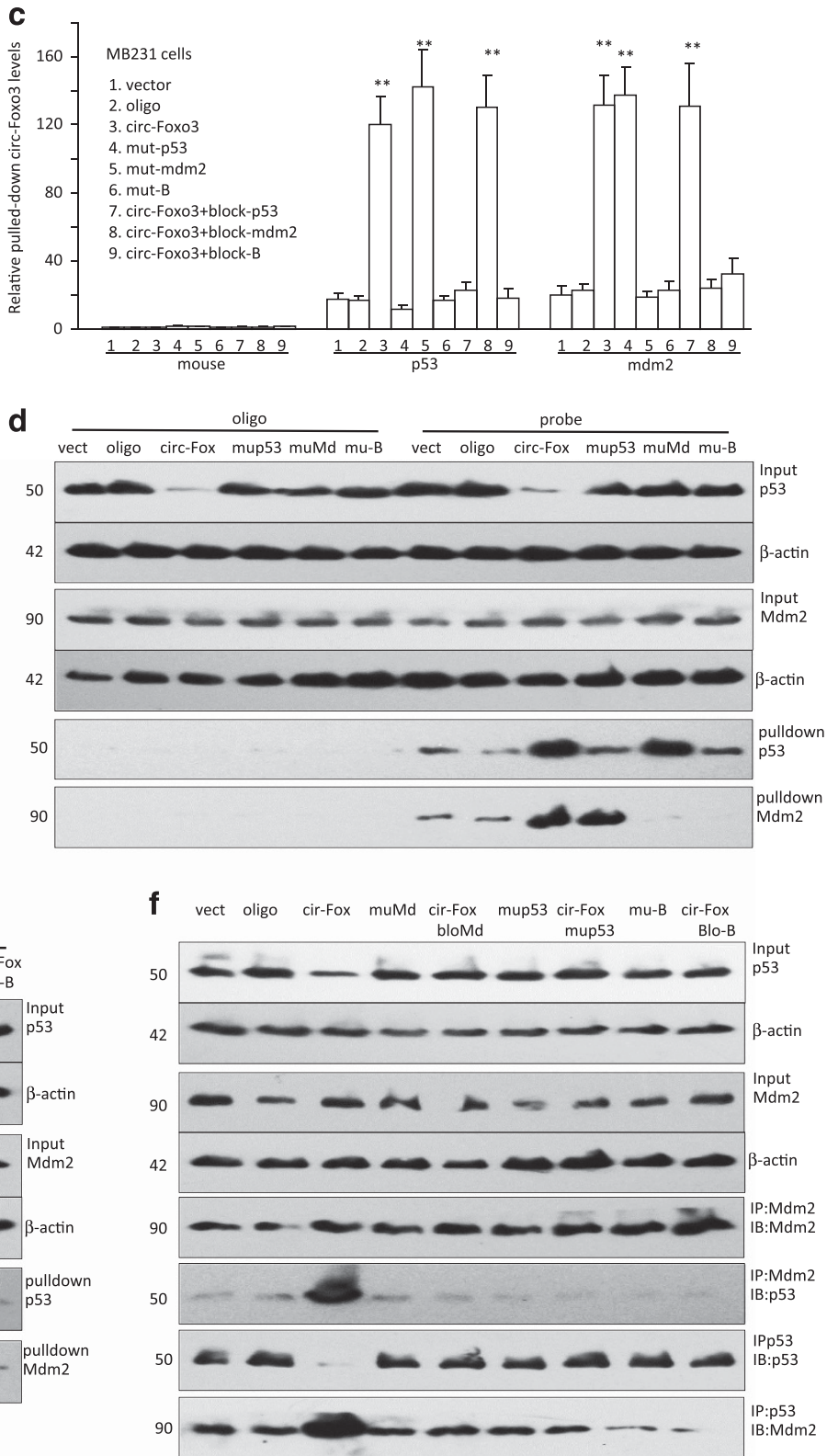

g

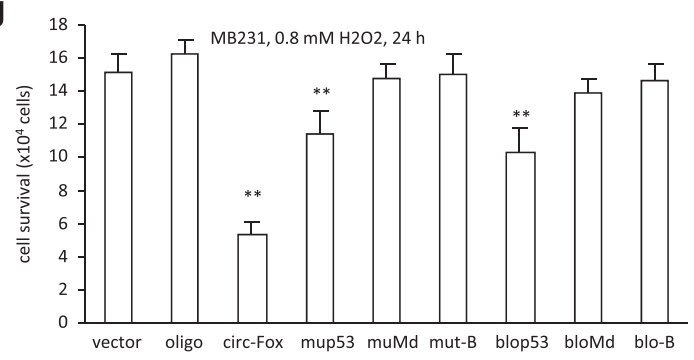

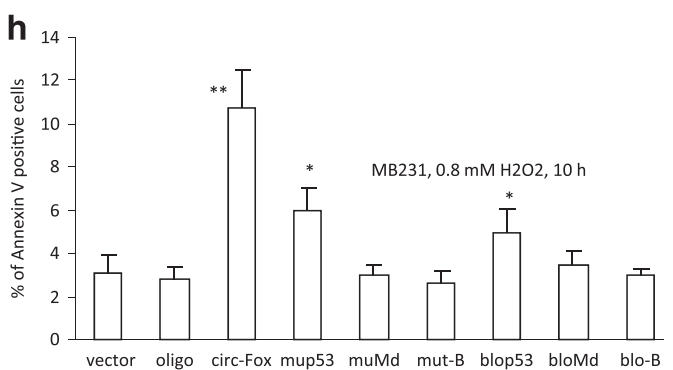


a

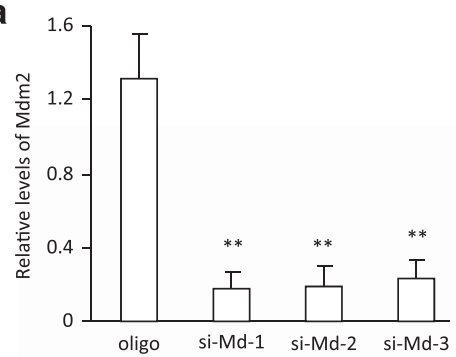

C

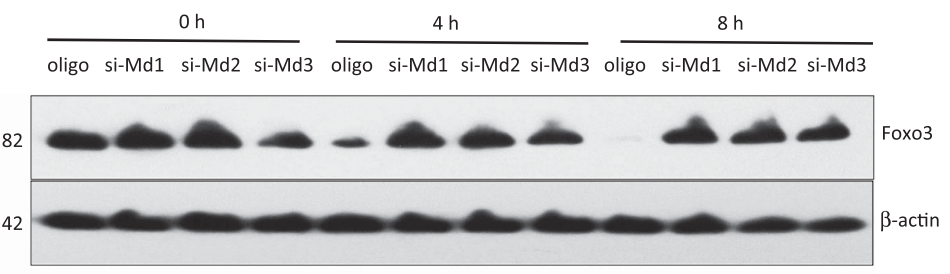

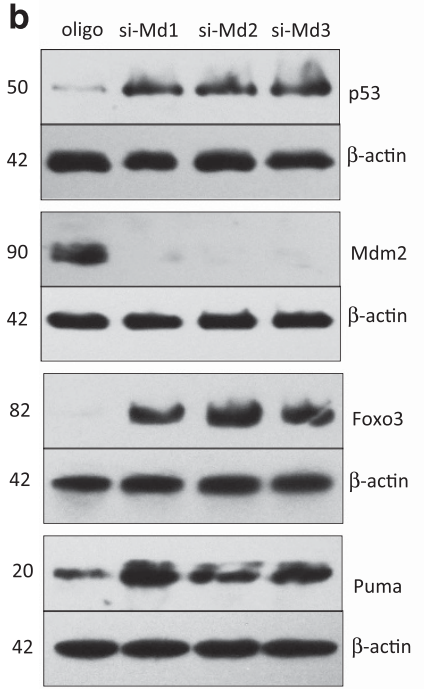

e

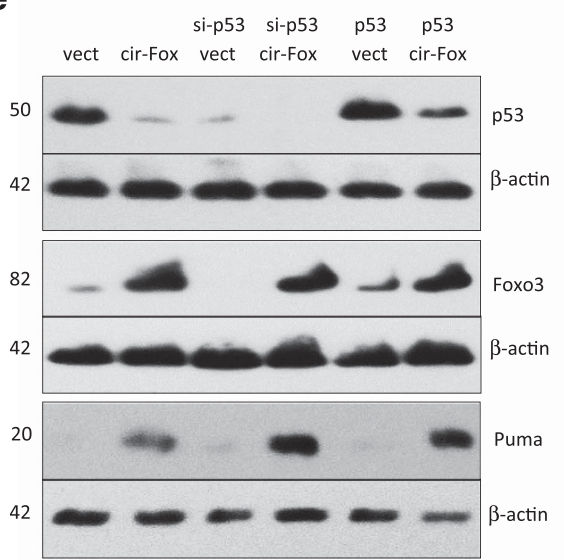

d
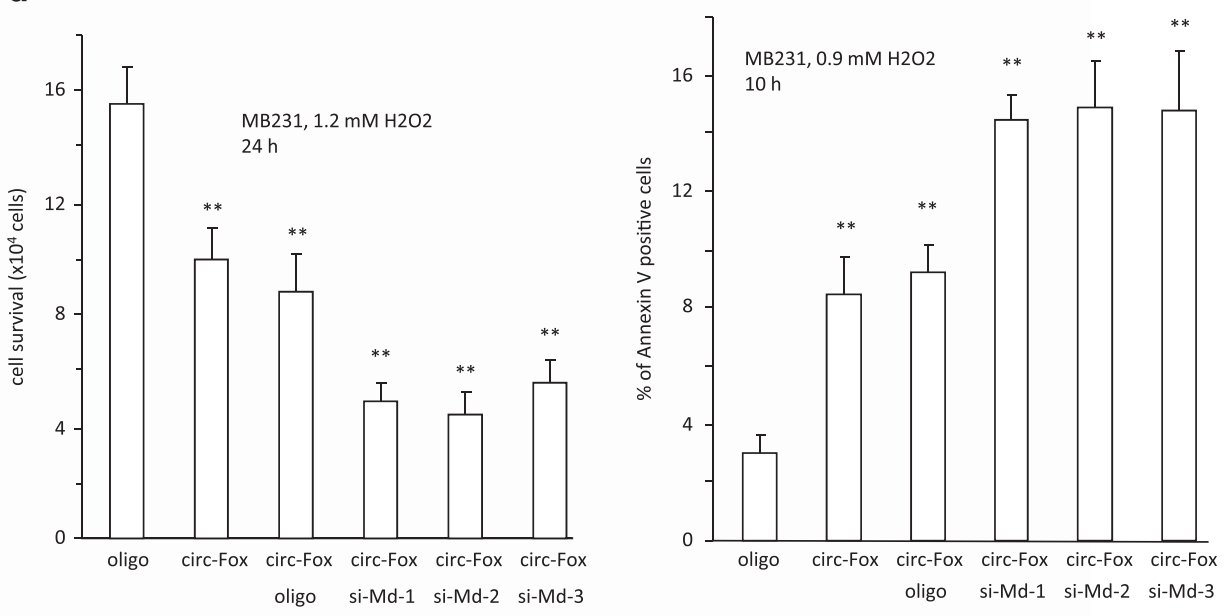

$\mathbf{f}$
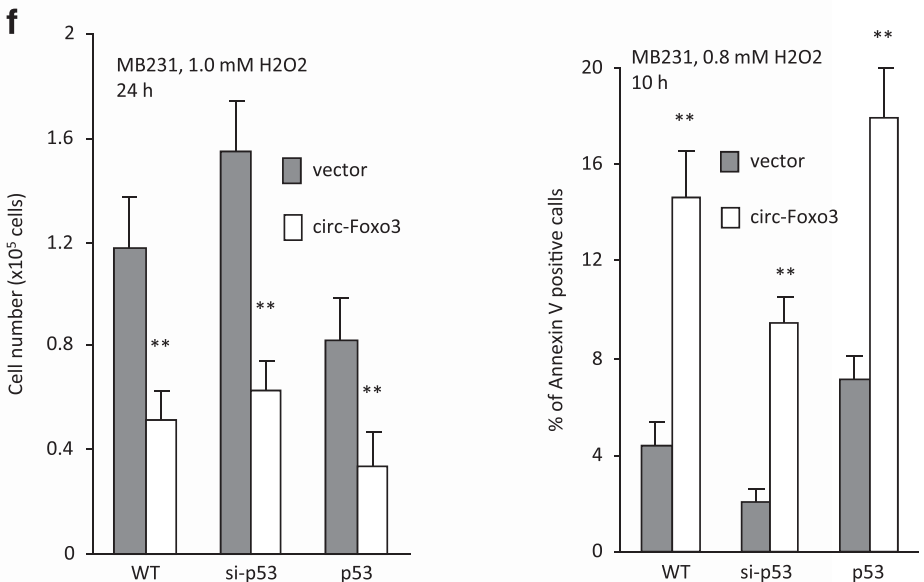

Figure 7 circ-Foxo3 function by modulating expression of p53 and Foxo3. (a) Real-time PCR showed that three siRNAs-targeting Mdm2 decreased Mdm2 levels in MB-231 cells transfected with these siRNAs. ${ }^{*} P<0.01$. Error bars, S.D. $(n=3)$. (b) Western blot showed that silencing Mdm2 enhanced expression of $p 53$, Foxo3 and Puma. (c) MB-231 cells transfected with Mdm2 siRNA or a control oligo were treated with $50 \mu \mathrm{M}$ Emetine for the time points as indicated. Silencing Mdm2 inhibited Fox03 degradation. (d) Left, silencing MDM2 decreased cell survival. ${ }^{* *} P<0.01$. Error bars, S.D. $(n=4)$. Right, silencing Mdm2 increased apoptosis which showed enhanced annexin-V staining ${ }^{\star *} P<0.01$. Error bars, S.D. $(n=4)$. (e) Western blot showed that transfection with circ-Fox03 enhanced Foxo3 and Puma expression with p53 silencing or p53 overexpression. (f) Left, transfection with circ-Fox03 repressed cell survival with p53 silencing or p53 overexpression. ${ }^{\star \star} P<0.01$. Error bars, S.D. $(n=4)$. Right, transfection with circ-Foxo3 increased cell apoptosis with p53 silencing or p53 overexpression. The cells were cultured in $0.9 \mathrm{mM} \mathrm{H} 2 \mathrm{O} 2$ for $10 \mathrm{~h}$, showing that expression of circ-Fox03 ${ }^{* \star} P<0.01$. Error bars, S.D. $(n=4)$

Foxo3 levels. Immunoprecipitation showed that more p53 and less Foxo3 were pulled down by the MDM2 precipitation in the circ-Foxo3-transfected cells, compared with the mock control (Figure 8a). In the MB-231 cells transfected with circ-Foxo3 siRNA, lower amounts of p53 and higher amounts of Foxo3 were pulled down by anti-MDM2 antibody (Figure 8b).

To confirm that circ-Foxo3 had a role in regulating MDM2's function in p53 ubiquitination, we employed the proteasome inhibitor MG-132 to block protein degradation. Although lower 
a

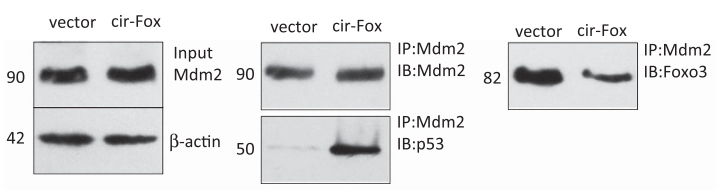

b

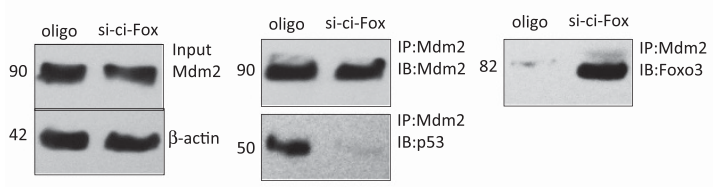

C
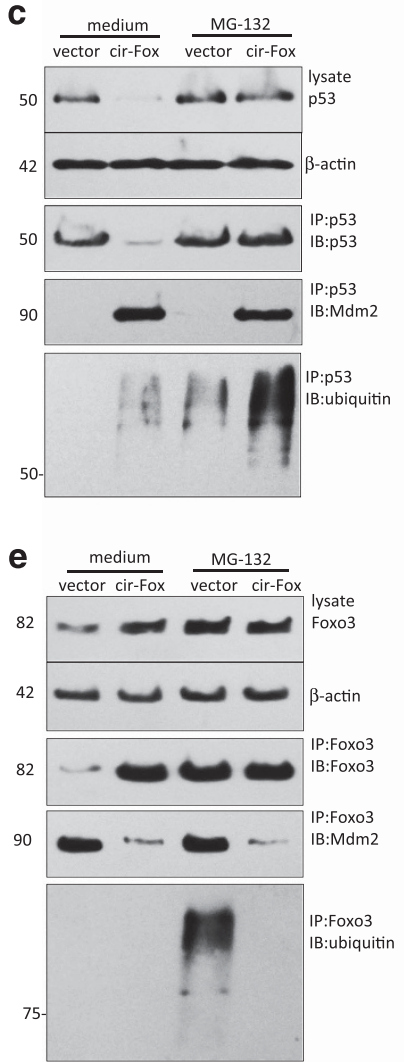

d $\frac{\text { medium }}{\text { oligo si-ci-Fox }} \frac{\text { MG-132 }}{\text { oligo si-ci-Fo }}$

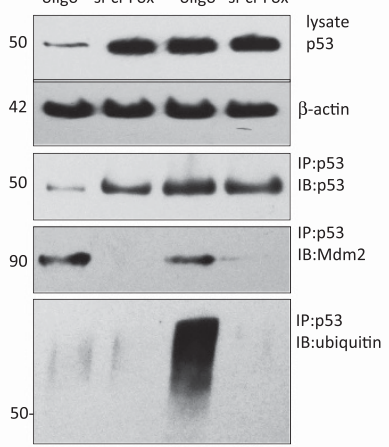

f

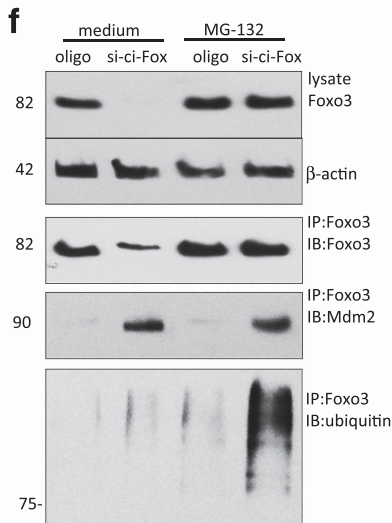

g

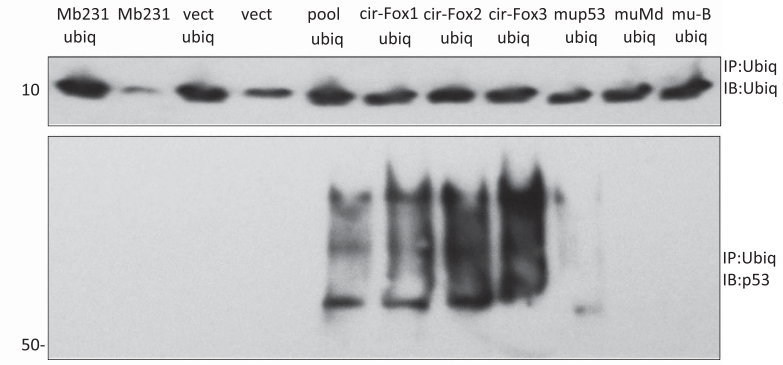

h

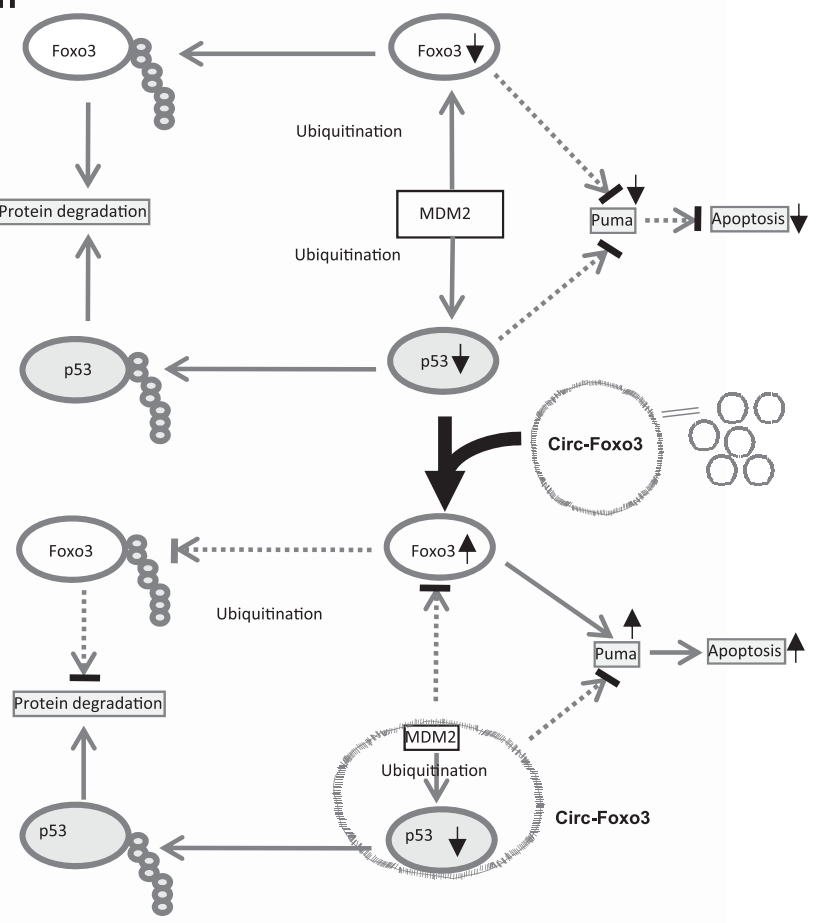

Figure 8 Expression of circ-Fox03 modulated the function of MDM2 in ubiquitination of p53 and Foxo3. (a) Circ-Foxo3 expression had no effect on MDM2 levels. MDM2 precipitation pulled down more p53 and less Fox03 in the circ-Fox03-transfected cells than in the mock control. (b) Transfection with circ-Foxo3 siRNA did not affect MDM2 expression. MDM2 precipitation pulled down more Fox03 but less p53 in the circ-Fox03 siRNA-transfected cells. (c) circ-Foxo3 transfection decreased p53 level. Treatment with $10 \mu \mathrm{M}$ MG-132 for $12 \mathrm{~h}$ abolished this effect. p53 precipitation pulled down more MDM2 and ubiquitin (detected with antibody against ubiquintin from Cell signalling, Cat No. 3933) in the circ-Fox03-transfected cells. (d) Transfection with circ-Fox03 siRNA increased p53 levels, which was abolished by treatment with MG-132. p53 precipitation pulled down less MDM2 and ubiquitin in the circ-Foxo3 siRNA-transfected cells. (e) Foxo3 precipitation pulled down less MDM2 and ubiquitin in the circ-Foxo3 transfected cells. (f) Fox03 precipitation pulled down more MDM2 and ubiquitin in the circ-Fox03 siRNA-transfected cells. (g) Cell lysates prepared from MB-231 cells co-transfected with ubiquintin and mock control, circ-Fox03, mup53, muMd or mu-B, were subject to precipitation with antibody against ubiquintin. Precipitation of ubiquintin pulled down p53 in the circ-Foxo3-overexpressing cell lines. (h) MDM2 acts as an E3 ubiquitin ligase, targeting both p53 and Foxo3 for degradation in proteasome. MDM2 is capable of poly-ubiquitinating p53 and Fox03. circ-Fox03 interacts with both MDM2 and p53, which enhances the interaction between MDM2 and p53, and further increases the poly-ubiquitination and degradation of p53. Another target protein of MDM2, Fox03, is free from being degraded. Thus, overexpression of circ-Fox03, decreases the interaction between Fox03 and MDM2, decreasing the amount of poly-ubiquitination and degradation of Fox03. Increased Fox03 then promotes expression of Puma, and enhances cell apoptosis

p53 levels were detected in the circ-Foxo3-transfected cells than in vector-transfected cells, p53 levels increased in both groups, when the cells were treated with MG-132, confirming the blockade of p53 (Figure 8c, upper). In both MG-132treated and untreated cells, the anti-p53 antibody could pulldown much higher levels of MDM2 and ubiquitin in the circFoxo3-transfected cells than those in the vector-transfected cells (Figure 8c, lower). This suggests increased uniquitination of p53 by MDM2. In contrast, siRNA transfection increased p53 levels when the cells were not treated with MG-132 (Figure 8d, upper), and anti-p53 antibody pulled down decreased levels of MDM2 and ubiquitin in the circ-Foxo3-transfected cells compared with the control (Figure 8d, lower). 
As expected, Foxo3 protein levels were much lower in the vector-transfected cells compared with the circ-Foxo3transfected cells. However, the levels of Foxo3 increased when both groups were treated with MG-132 (Figure 8e, upper). Anti-Foxo3 antibody pulled down hardly detectable MDM2 and ubiquitin in the circ-Foxo3-transfected cells (Figure 8e, upper), suggesting dissociation of Foxo3 and MDM2 in the presence of circ-Foxo3. Transfection with circ-Foxo3 siRNA increased Foxo3 antibody pulling-down MDM2 and ubiquitin (Figure 8f), suggesting re-association of Foxo3 with MDM2 when the endogenous circ-Foxo3 was silenced. We used an ubiquitin expression construct (Addgene, Cambridge, MA, USA, 18712 HA-Ubiquitin) and co-transfected it with circ-Foxo3. Precipitation with antibody against ubiquintin confirmed that $\mathrm{p} 53$ was precipitated when the cells were transfected with circ-Foxo3 (Figure 8g). However, mutations of circ-Foxo3 abolished ubiquitination of p53, indicating the essential role of circ-Foxo3 in mediating p53Mdm2 interaction.

\section{Discussion}

In this study, we detected significantly lower levels of circ-Foxo3 in breast carcinoma biopsies and in cancer cell lines. circ-Foxo3 expression increased when cancer cells underwent apoptosis. Transfection of a circ-Foxo3 expression construct triggered stress-induced apoptosis and inhibited tumor growth. In the transfected cells, Foxo3 levels increased but p53 levels decreased. circ-Foxo3 promoted MDM2induced p53 degradation, avoiding MDM2 to induce Foxo3 ubiquitination because both MDM2 and p53 could bind circ-Foxo3 but not Foxo3 linear mRNA. Owing to the large size of circ-Foxo3, we selected the most important nucleotides at the junction of circularization and its flanking regions for binding analysis, as these regions have a critical role in circularization of circ-Foxo3.

Expression of p53 is crucial in multi-cellular organisms, where it functions as a tumor suppressor and prevents cancer formation. ${ }^{20}$ At a basal level, p53 is continually degraded, a process associated with MDM2-binding. When p53 expression is induced and stabilized, it also induces MDM2 expression. By binding to p53, MDM2 prevents p53's action and transports it from nucleus to cytosol, where MDM2 acts as E3 ubiquitin ligase and covalently links ubiquitin to p53, marking p53 for degradation by the proteasome. It has been known that p53 is the key target of MDM2. Our study introduces a new mechanistic understanding to the homeostatic balance between p53 and MDM2. We found that circFoxo3 could interact with both p53 and MDM2, enhancing the binding of p53 and MDM2 and facilitating the addition of ubiquitin to $\mathrm{p} 53$ by MDM2. To confirm the increased action of MDM2 on p53 ubiquitination by circ-Foxo3, we employed siRNA to silence endogenous circ-Foxo3. In the circ-Foxo3 siRNA-transfected cells, the total levels of p53 increased, whereas MDM2 level was not affected.

It has been known that mutant p53 fails to induce MDM2 expression, resulting in p53 accumulation. In addition, MDM2 cannot bind mutant p53 as effectively as wild-type p53. This would decrease the translocation of mutant p53 from nucleus to cytosol, which is an essential step for p53 degradation.
Mutant p53 would inhibit the stability and function of wild-type p53. ${ }^{21}$ The interaction of circ-Foxo3-p53-MDM2 may add an additional layer of action for MDM2 to interact with mutant p53, leading to the ubiquitination of the mutant p53. This may raise the possibility for a protective mechanism by which mutant p53 can be removed from the cells, and may provide the basis for a future line of study. In the p53 wild-type cells, the interaction of circ-Foxo3-p53-MDM2 may relieve Foxo3 ubiquitination. Foxo3 typically functions as a trigger for apoptosis through upregulation of genes necessary for cell death, such as Puma. ${ }^{22}$ In our results, we showed that Puma levels were upregulated in the cells transfected with circ-Foxo3, producing an additional mechanism for cell apoptosis induction. Thus, in both p53 mutant cells and p53 wild-type cells, circ-Foxo3 could form the complex with MDM2 and either mutant p53 or wildtype p53, leading to induction of tumor cell death. Since p53 mutation represents $50 \%$ cancers and in many cancer cell lines including the cell lines MDA-MB-231 and 4T1 used in this study, our results that circ-Foxo3 could induce apoptosis of p53 mutant and wild-type cells are of clinically significance, and circ-Foxo3 may be developed as an agent for cancer intervention.

In summary, we showed that circ-Foxo3 could bind both p53 and MDM2 (Figure 8h). MDM2 is capable of poly-ubiquitinating p53 and Foxo3, and it has been shown that p53 and Foxo3 are both downregulated by MDM2 in a proteasome-dependent manner. ${ }^{23}$ Thus MDM2 has an important role in repressing apoptosis by suppressing p53, Foxo3, and their downstream molecule Puma. The dual role of circ-Foxo3 in modulating the poly-ubiquitination function of MDM2 is mainly related to the strong tendency of circ-Foxo3 to bind MDM2, and the interaction between circ-Foxo3 and p53. This further enhances the function of MDM2 in modulating poly-ubiquitination of p53. Foxo3, another target protein of MDM2, which has no tendency to bind circ-Foxo3, is thus freed from this degradation. In conclusion, we found that overexpression of circ-Foxo3 decreased the interaction between Foxo3 and MDM2, and repressed the function of MDM2 in modulating poly-ubiquitination of Foxo3. This, in turn, increased Foxo3 activity, promoting Puma expression and cell apoptosis.

\section{Materials and Methods}

Materials. The monoclonal antibodies against p53 and MDM2 were purchased from Santa Cruz (Santa Cruz County, CA, USA). The monoclonal antibodies against Fox03 and Puma were obtained from Cell Signalling (Danvers, MA, USA). Horseradish peroxidase-conjugated goat anti-mouse IgG and horseradish peroxidase-conjugated goat anti-rabbit IgG were obtained from Bio-Rad (Hercules, CA, USA). RNA and DNA extract kits, RNA RT and PCR kits, were obtained from Qiagen (Venlo, Netherlands). NorthernMAX kit was from Ambion (Waltham, MA, USA). Biotin Chromogenic Detection kit was from Thermo Scientific (Waltham, MA, USA). Annexin-V-FITC apoptosis detection kit was obtained from Biovision (Zurich, Switzerland). Protein A-Sepharose 4B Conjugate and Streptavidin C1 magnetic beads were obtained from Invitrogen (Waltham, MA, USA). MG-132 and Emetine dihydrochloride hydrate were obtained from Sigma-Aldrich (St. Louis, MO, USA).

Constructs and primers. A construct of mouse circular RNA Fox03 (circFox03) was generated by us. ${ }^{10}$ All primer sequences used are listed in Supplementary Figure S9b. The anti-circ-Foxo3 siRNA, DNA oligo probes against endogenous or ectopic expression of circ-Fox03, labeled with biotin or Cy5 were obtained from Integrated DNA Technologies (Coralville, IA, USA) (Supplementary Figure S9c). 
Cell survival and apoptosis assays. The procedures for cell survival and apoptosis analysis were performed as described. ${ }^{8,24}$

Western blot analysis. Protein analysis on western blotting was performed as described. ${ }^{25}$

RT-PCR and real-time PCR. In total, $2 \times 10^{6}$ cells were harvested, and total RNAs were isolated with Qiagen RNeasy mini kit. Two micrograms of total RNAs were used to synthesize CDNA, a portion of which ( $1 \mu \mathrm{l}$, equal to $0.2 \mu \mathrm{g}$ CDNA) was used in a PCR with two appropriate primers. Real-time PCR was performed with miScriptSYBR GreenPCR Kit (Qiagen) using $1 \mu \mathrm{l}$ cDNA as the template as described. ${ }^{26}$

Immunoprecipitation assay. In total, $10^{7}$ cells were harvested and washed in ice-cold phosphate-buffered saline followed by lysis in $1 \mathrm{ml}$ lysis buffer. Equal amounts of protein were incubated with $5 \mu \mathrm{g}$ primary antibodies and $40 \mu \mathrm{l}$ of $50 \%$ slurry of protein A-Sepharose at $4{ }^{\circ} \mathrm{C}$ for $4 \mathrm{~h}$. Each pellet was washed three times with PBS and resuspended in $2 \times$ Laemmli buffer $(0.125 \mathrm{M}$ Tris-Cl, $4 \%$ SDS, $20 \%$ glycerol, 10\% 2-mercaptoethanol, 0.004\% bromophenol blue, pH 6.8), followed by western blot analysis.

RNA binding assays. The methods for protein precipitating RNA and RNA pull-down proteins were performed as described. ${ }^{11}$

Northern blot. RNA was isolated with RNA extract kit and northern blot analysis was performed with Northern blot kit (Ambion). In brief, the preparation of total RNA $(30 \mu \mathrm{g})$ was denatured in formaldehyde and then subject to electrophoresis in a 1\% agarose-formaldehyde gel. The RNA was then transferred to a Hybond-N+nylon membrane (Amersham, Amersham, UK) and hybridized to biotin-labeled DNA probes. Biotin Chromogenic Detection kit (Thermo Scientific) was used to detect the bound RNA molecule.

Tumorigenicity, mouse survival, and immunostaining. Tumorigenesis was performed as described previously. ${ }^{25,27}$ All animal experiments were conducted according to the guideline approved by the Animal Care Committee at Sunnybrook Research Institute (Protocol Number, AUP 15-076). Animals were kept in the Animal Core Facility of Sunnybrook Research Institute for one week before use. In brief, 6-week-old regular Balb/c mice were injected subcutaneously with $4 \mathrm{~T} 1$ cells $\left(2 \times 10^{6}\right.$ cells in $\left.100 \mu l\right)$. Twelve days after the injection, the mice were killed and tumors were removed. Tumor volume $(V)$ was estimated using a caliper by measuring the length $(L)$ and width $(W)$, where $V=\left(L \times W^{2}\right) / 2$. In the second injection, $5 \times 10^{5}$ cells (in $100 \mu l$ ) were injected. 18 days after the injection, the mice were killed and tumors were removed.

In separate experiments, CD-1 nude mice were injected subcutaneously with MDA-MB-231 cells $\left(1 \times 10^{7}\right.$ cells in $\left.100 \mu \mathrm{l}\right)$. Fourteen days after the injection, the mice were killed and tumors were removed to determine the sizes of the tumors. Immunostaining was performed as described. ${ }^{26}$

In survival assay, 4-week-old C57 mice were randomly divided into three groups: B16+control vector, B16+circ-Fox03 and circ-Fox03 transfected B16. Each group had 15 mice. The B16+vector and B16+circ-Fox03 groups were injected intraperitoneally with B16 cells $\left(2 \times 10^{4}\right.$ cells/mouse). Next day after cell implantation, circ-Fox03 plasmid-PEG-Au NP complexes were injected intraperitoneally at a dose of $50 \mu \mathrm{g}$ plasmid/mouse. This was repeated every three days until the experiment was completed. The control group was mice injected with $0.9 \%$ sodium chloride at the same volume on the same schedule. The circ-Foxo3-transfected B16 group mice were injected intraperitoneally with circ-Foxo3 transfected B16 cells $\left(2 \times 10^{4}\right.$ cells/ mouse), and were injected with $0.9 \%$ sodium chloride at the same volume on the same schedule as a positive control. Synthesis of the deliver complexes (plasmid -PEG-Au NP) was performed as previously described. In brief, $750 \mu \mathrm{g}$ circ-Fox03 plasmid was dissolved in $800 \mu$ l of RNase-free water. The mPEGSH (PG1-TH-2k, Nanocs) was mixed with plasmid (1:20 molar ratio). Then 10-nm gold nanoparticles (Au NP, Cytodiagnostics, Burlington, Ontario, Canada) were mixed with the plasmid at a weight ratio of $1: 20$. The mixture was gently shaken at $60^{\circ} \mathrm{C}$ for 30 min and transferred into a syringe.

Docking simulations, contact maps, and identification of binding site residues. To determine the possible interaction of circ-Fox03 with MDM2 (RING-finger domain) and p53 (C-terminal regulatory domain), we employed the protein-RNA docking analysis tool NPDOCK Server ${ }^{28}$ and generated 20000 models. NPDOCK Server combines GRAMM for global macromolecular docking, scoring with a statistical potential, clustering followed by refinement of best scored docked complexes from three biggest clusters. Residue-level resolution contact maps of circ-Fox03-MDM2 (RING-finger domain) and circ-Fox03-p53 (C-terminal regulatory domain) docked complexes were determined using RNAmap2D as described. ${ }^{29}$ The contact distances between $\mathrm{C} \alpha$ atoms of protein residues and $05^{\prime}$ atoms of RNA strands were computed. Two residues were considered to be in contact when their 05'-C $\alpha$ distance is $<10 \AA$. Distance-based approach was used to identify the binding site residues/nucleotides for the protein-RNA complexes using a specific cutoff value. Two atoms (one in RNA and another in protein) were considered to be interacting with each other if the distance between them was $<3.5 \AA$.

Statistical analysis. All experiments were performed in triplicate or as indicated in the experiments and numerical data were subject to independent sample $t$-test. The levels of significance were set at ${ }^{*} P<0.05$ and ${ }^{*} P<0.01$.

\section{Conflict of Interest}

The authors declare no conflict of interest.

Acknowledgements. This work was supported by $\mathrm{ClHR}$ Bridge Fund (PJT149083) to BBY, National Natural Science Foundation of China (NSFC; 81472454) to LF, and a Career Investigator Award (Cl 7418) from the Heart and Stroke Foundation of Ontario to BBY. WWD is supported by a Postdoctoral Fellowship from the Breast Cancer Foundation of Ontario.

1. Jeck WR, Sharpless NE. Detecting and characterizing circular RNAs. Nat Biotechnol 2014; 32: 453-461.

2. Haimovich G, Medina DA, Causse SZ, Garber M, Millan-Zambrano G, Barkai O et al. Gene expression is circular: factors for mRNA degradation also foster mRNA synthesis. Cell 2013; 153: $1000-1011$.

3. Wilusz JE, Sharp PA. Molecular biology. A circuitous route to noncoding RNA. Science 2013; 340: $440-441$

4. Westholm JO, Miura P, Olson S, Shenker S, Joseph B, Sanfilippo P et al. Genome-wide analysis of drosophila circular RNAs reveals their structural and sequence properties and age-dependent neural accumulation. Cell Rep 2014; 9: 1966-1980.

5. Zhang XO, Wang HB, Zhang Y, Lu X, Chen LL, Yang L. Complementary sequence-mediated exon circularization. Cell 2014; 159: 134-147.

6. Zhang Y, Zhang XO, Chen T, Xiang JF, Yin QF, Xing YH et al. Circular intronic long noncoding RNAs. Mol Cell 2013; 51: 792-806.

7. Hansen TB, Jensen TI, Clausen BH, Bramsen JB, Finsen B, Damgaard CK et al. Natural RNA circles function as efficient microRNA sponges. Nature 2013; 495: 384-388.

8. Yang W, Du WW, Li X, Yee AJ, Yang BB. Foxo3 activity promoted by non-coding effects of circular RNA and Foxo3 pseudogene in the inhibition of tumor growth and angiogenesis. Oncogene 2016; 35: 3919-3931.

9. Li Z, Huang C, Bao C, Chen L, Lin M, Wang X et al. Exon-intron circular RNAs regulate transcription in the nucleus. Nat Struct Mol Biol 2015; 22: 256-264.

10. Du WW, Yang W, Chen Y, Wu ZK, Foster FS, Yang Z et al. Foxo3 circular RNA promotes cardiac senescence by modulating multiple factors associated with stress and senescence responses. Eur Heart J 2016 (in press).

11. Du WW, Yang W, Liu E, Yang Z, Dhaliwal P, Yang BB. Foxo3 circular RNA retards cell cycle progression via forming ternary complexes with p21 and CDK2. Nucleic Acids Res 2016; 44: 2846-2858.

12. Elenbaas B, Dobbelstein M, Roth J, Shenk T, Levine AJ. The MDM2 oncoprotein binds specifically to RNA through its RING finger domain. Mol Med 1996; 2: 439-451.

13. Yoshida $Y$, Izumi $H$, Torigoe $T$, Ishiguchi $H$, Yoshida $T$, Itoh $H$ et al. Binding of RNA to $p 53$ regulates its oligomerization and DNA-binding activity. Oncogene 2004; 23: 4371-4379.

14. Myatt SS, Lam EW. The emerging roles of forkhead box (Fox) proteins in cancer. Nat Rev Cancer 2007; 7: 847-859

15. Cho EC, Kuo ML, Liu X, Yang L, Hsieh YC, Wang J et al. Tumor suppressor FOXO3 regulates ribonucleotide reductase subunit RRM2B and impacts on survival of cancer patients. Oncotarget 2014; 5: 4834-4844.

16. Anderson MJ, Viars CS, Czekay S, Cavenee WK, Arden KC. Cloning and characterization of three human forkhead genes that comprise an FKHR-like gene subfamily. Genomics 1998; 47: 187-199.

17. Hofacker IL, Stadler PF. Memory efficient folding algorithms for circular RNA secondary structures. Bioinformatics 2006; 22: 1172-1176.

18. Zuker M. Mfold web server for nucleic acid folding and hybridization prediction. Nucleic Acids Res 2003; 31: 3406-3415. 
19. Popenda M, Szachniuk M, Antczak M, Purzycka KJ, Lukasiak P, Bartol N et al. Automated 3D structure composition for large RNAs. Nucleic Acids Res 2012; 40: e112.

20. Surget S, Khoury MP. Bourdon JC. Uncovering the role of $p 53$ splice variants in human malignancy: a clinical perspective. Onco Targets Ther 2013; 7: 57-68.

21. Bullock AN, Henckel J, DeDecker BS, Johnson CM, Nikolova PV, Proctor MR et al. Thermodynamic stability of wild-type and mutant p53 core domain. Proc Natl Acad Sci USA 1997; 94: 14338-14342.

22. Ekoff M, Kaufmann T, Engstrom M, Motoyama N, Villunger A, Jonsson Jl et al. The BH3-only protein Puma plays an essential role in cytokine deprivation induced apoptosis of mast cells. Blood 2007; 110: 3209-3217.

23. Haupt $Y$, Maya R, Kazaz A, Oren M. Mdm2 promotes the rapid degradation of p53. Nature 1997; 387: 296-299.

24. Wu Y, Chen L, Cao L, Sheng W, Yang BB. Overexpression of the C-terminal PG-M/versican domain impairs growth of tumor cells by intervening in the interaction between epidermal growth factor receptor and beta1-integrin. J Cell Sci 2004; 117: 2227-2237.

25. Rutnam ZJ, Du WW, Yang W, Yang X, Yang BB. The pseudogene TUSC2P promotes TUSC2 function by binding multiple microRNAs. Nat Commun 2014; 5: 2914.

26. Shan SW, Lee DY, Deng Z, Shatseva T, Jeyapalan Z, Du WW et al. MicroRNA MiR-17 retards tissue growth and represses fibronectin expression. Nat Cell Biol 2009; 11: 1031-1038.

27. Yang X, Du WW, Li H, Liu F, Khorshidi A, Rutnam ZJ et al. Both mature miR-17-5p and passenger strand miR-17-3p target TIMP3 and induce prostate tumor growth and invasion. Nucleic Acids Res 2013; 41: 9688-9704.

28. Tuszynska I, Magnus M, Jonak K, Dawson W, Bujnicki JM. NPDock: a web server for protein-nucleic acid docking. Nucleic Acids Res 2015; 43: W425-W430.

29. Pietal MJ, Szostak N, Rother KM, Bujnicki JM. RNAmap2D - calculation, visualization and analysis of contact and distance maps for RNA and protein-RNA complex structures. BMC Bioinformatics 2012; 13: 333.

Supplementary Information accompanies this paper on Cell Death and Differentiation website (http://www.nature.com/cdd) 